\title{
SEARCHES FOR DIJET RESONANCES AT HADRON COLLIDERS
}

\author{
ROBERT M. HARRIS \\ Fermilab \\ Batavia, Illinois 60510 \\ United States of America \\ rharris@fnal.gov \\ KONSTANTINOS KOUSOURIS* \\ CERN \\ Geneva \\ Switzerland \\ Konstantinos.Kousouris@cern.ch
}

\begin{abstract}
We review the experimental searches for new particles in the dijet mass spectrum conducted at the CERN $S \bar{p} p S$, the Fermilab Tevatron Collider, and the CERN Large Hadron Collider. The theory of the QCD background and new particle signals is reviewed, with emphasis on the choices made by the experiments to model the background and signal. The experimental techniques, data, and results of dijet resonance searches at hadron colliders over the last quarter century are described and compared. Model independent and model specific limits on new particles decaying to dijets are reviewed, and a detailed comparison is made of the recently published limits from the ATLAS and CMS experiments.
\end{abstract}

\section{Introduction}

Experiments at hadron colliders have used the dijet mass spectrum to search for new particles beyond the standard model. At the CERN $S \bar{p} p S$, the Fermilab Tevatron Collider, and the CERN Large Hadron Collider, with each successive advance in collision energy and integrated luminosity, progressively more energetic collisions of the partons in the incoming hadrons are produced and observed. Each machine in its time has therefore probed the highest masses of dijet resonances: new particles that decay into two partons, giving two jets in the final state. The simple process the experiments have searched for is the $s$ - channel production and decay of dijet resonances shown in Fig. 1]

Here we review these experimental searches, their techniques, data, results, and limits on new particles. In section 2 we review the theory of the QCD background and the models of new particle signals. In section 3 we review the experiments from each collider in chronological order. In section 3.1 we review how each experiment

*Also affiliated with Fermilab during this review. 


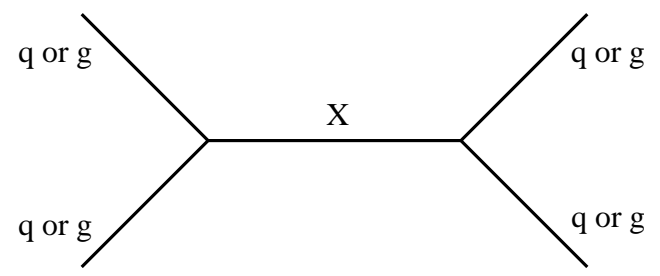

Fig. 1. Diagram of dijet resonance in the $s-$ channel. The initial state and final states contain two partons (quarks, anti-quarks or gluons) and the intermediate state contains a resonance $X$.

modeled the resonance shapes as a function of the dijet mass. In section 3.2 we review the data of each experiment and how each experiment modeled the QCD background. In section 3.3 we review the limits on dijet resonance masses published by each experiment, discussing the experimental uncertainties, statistical procedures, and the cross section assumed for each model. In section 4 we conclude with a few observations. Also, in Appendix A, we include details of the cross-section calculations for axigluons and excited quarks by ATLAS and CMS, which are necessary to understand the mass limits on these models from the two experiments.

\section{Theory}

In this section we present the fundamental ingredients of the theory, which are necessary for the better understanding of the experimental searches presented in this review. In Section 2.1 we describe some basic features of Quantum Chromodynamics (QCD), and in Section 2.2 we present the theoretical models that predict partonic resonances and are quoted in the experimental searches. It should be noted, that the purpose of this section is not to give all the details of the models presented, but rather an overview of their features.

\subsection{Elements of $Q C D$}

\subsubsection{The QCD Lagrangian}

Quantum Chromodynamics is the gauge field theory of the strong interaction between particles that carry the color degree of freedom. The underlying symmetry group is the $\mathrm{SU}(3)_{C}$, which makes QCD a non-Abelian theory. The profound implication of this property of QCD is that the gauge mediators (gluons) are colored and thus self interacting. The QCD Lagrangian is written as:

$$
\mathcal{L}_{Q C D}=\sum_{i} \bar{q}_{i, a}\left(i \gamma^{\mu} \partial_{\mu} \delta_{a b}-g_{s} \gamma^{\mu} t_{a b}^{C} G_{\mu}^{C}-m_{i} \delta_{a b}\right) q_{i, b}-\frac{1}{4} F_{\mu \nu}^{A} F^{\mu \nu, A},
$$

where $q_{i, a}$ represents the quark spinor of flavor $i$ and color $a=1 \rightarrow 3, G_{\mu \nu}^{A}$ is the gluon field associated with the generator $t_{a b}^{A}(A=1 \rightarrow 8), g_{s}$ is the gauge coupling, and $F_{\mu \nu}^{A}$ is the gluon field tensor:

$$
F_{\mu \nu}^{A}=\partial_{\mu} G_{\mu}^{A}-\partial_{\nu} G_{\nu}^{A}-g_{s} f_{A B C} G_{\mu}^{B} G_{\nu}^{C}
$$


The structure constants $f_{A B C}$ satisfy the relation:

$$
\left[t^{A}, t^{B}\right]=i f_{A B C} t^{C} \text {. }
$$

The non-Abelian nature of QCD leads to two remarkable features: the confinement and the asymptotic freedom. As a result of the confinement, only color-singlet states can be directly observed, which means that quarks and gluons cannot be found free. The asymptotic freedom is the property where the running strong coupling constant decreases with increasing momentum transfer between the strongly interacting particles. This in turn means, that the hard-scattering of quarks and gluons can be described in a perturbative way.

\subsubsection{Formation of jets}

Because of the confinement, partons cannot be detected free. Instead, the experimental signatures of quarks and gluons are the jets. A jet is a "spray" of highly collimated particles, primarily hadrons, but also photons and leptons. A jet is a not uniquely defined object, but the output of a well-defined mathematical rule (clustering algorithm), which clusters the jet constituents, according to their kinematic properties. This procedure is based upon the features of QCD, which describe the transformation of a parton to a set of observable particles. The jet-formation steps are the following:

- Parton branching: each parton, whether a gluon or a quark, has a finite probability to split into two partons, which are emitted in small angles with respect to the direction of the initial parton. One feature of the parton branching is that the probability depends on the color factor related to the type of the involved partons. For gluon $\rightarrow$ gluon, gluon, gluon $\rightarrow$ quark, antiquark, and quark $\rightarrow$ gluon, quark splittings, the color factors are $C_{g g}=3, C_{q q}=\frac{4}{3}$, and $C_{q g}=\frac{1}{2}$ respectively. As a result, gluons systematically shower more than quarks. Another implication of parton branching in small angles is that throughout the process, partons are produced close to the direction of the initial partons, which results in a high degree of collimation of the final hadrons. It should be noted, that the parton branching is a perturbative procedure, which can be re-summed to all orders of the perturbation series, under certain assumptions.

- Hadronization: when the parton shower has evolved long enough, the energy of the partons is reduced, such that low-momentum transfer occur. In these conditions, the parton interactions become non-perturbative, and the phase of hadronization begins. During the hadronization, partons are combined into color-singlet states, thus forming the hadrons. While the hadronization procedure cannot be described perturbatively, the local parton-hadron duality ensures that the flow of quantum numbers at the hadron level, follows approximately the corresponding flow at parton level. 
- Underlying event $\mathbb{G}$ out-of-cone showering: the term underlying event in hadron collisions is used to describe the activity not related to the hard scattering, for example due to multiple parton interactions happening simultaneously. Since the definition of jets involve the clustering of hadrons which are sufficiently correlated, it can happen that particles originating from the soft interactions are clustered together with those coming from the hard-scattered parton shower. In the opposite direction, partons from the initial shower can be emitted in relatively large angles, and the associated hadrons may not be clustered in the resulting jets. This effect is commonly known as out-of-cone showering.

Despite the fact that the formation of jets is a complicated effect, certain conclusions can be drawn: to first approximation, the kinematical properties of a jet are the same as those of the original parton. However, the various effects involved, introduce an intrinsic resolution of the hadronic jet properties with respect to the parton properties.

\subsubsection{Kinematics of two-parton scattering}

Before the details of the strong interaction dynamics are discussed, it is useful to present the kinematical properties of a two-to-two parton scattering. In the topology of the $1+2 \rightarrow 3+4$ scattering, some general kinematic relations hold, regardless of the details of the interaction. The Mandelstam variables of the process are defined as $\hat{s}=\left(p_{1}+p_{2}\right)^{2}, \hat{t}=\left(p_{1}-p_{3}\right)^{2}$, and $\hat{u}=\left(p_{2}-p_{3}\right)^{2}$, where $p_{i}$ are the four-momenta of the partons. For massless partons, the Mandelstam variables satisfy the relation $\hat{s}+\hat{t}+\hat{u}=0$ and two of those can be expressed as a function of the third one and the scattering angle $\theta^{*}$ in the center-of-mass frame:

$$
\hat{t}=-\frac{1}{2} \hat{s}\left(1-\cos \theta^{*}\right), \quad \hat{u}=-\frac{1}{2} \hat{s}\left(1+\cos \theta^{*}\right) .
$$

The rapidities of the outgoing partons, in the center-of-mass frame, are opposite $\left( \pm y^{*}\right)$, due to transverse momentum conservation, and related to the scattering angle:

$$
\cos \theta^{*}=\tanh y^{*} .
$$

The Mandelstam variable $\hat{s}$ can be expressed in terms of the outgoing partons transverse momentum $p_{T}$ and $y^{*}$ :

$$
\hat{s}=4 p_{T}^{2} \cosh ^{2} y^{*} .
$$

In the laboratory frame, the rapidities $y_{3,4}$ of the outgoing partons are related to the rapidity of the center-of-mass frame $\bar{y}$ and to $y^{*}$ as:

$$
\bar{y}=\frac{y_{3}+y_{4}}{2}, y^{*}=\frac{y_{3}-y_{4}}{2} .
$$

${ }^{\mathrm{a}} y=\frac{1}{2} \ln \left(\frac{E+p_{z}}{E-p_{z}}\right)$ 
From the relations above, one can express the scattering angle at the center-of-mass frame as a function of the rapidities of the scattered partons at the laboratory frame:

$$
\cos \theta^{*}=\tanh \left(\frac{y_{3}-y_{4}}{2}\right)
$$

Each initial parton is carrying a fraction $x$ of the hadron momentum and the invariant mass of the two-parton system is expressed as:

$$
M^{2}=\hat{s}=x_{1} x_{2} s,
$$

where $x_{1,2}$ are the momentum fractions of the interacting partons and $\sqrt{s}$ is the colliding energy of the hadrons:

$$
x_{1}=\frac{2 p_{T}}{\sqrt{s}} \cosh y^{*} e^{\bar{y}}, x_{2}=\frac{2 p_{T}}{\sqrt{s}} \cosh y^{*} e^{-\bar{y}} .
$$

Following from the relation above, the rapidity of the center-of-mass frame $\bar{y}$ can be expressed as a function of the momentum fractions:

$$
\bar{y}=\frac{1}{2} \ln \frac{x_{1}}{x_{2}}
$$

\subsubsection{Partonic cross sections}

The dynamics of the hard scatter of colliding hadrons is approximately described as a two-to-two process between massless partons. Because of the different structure and color factors of the interaction between the parton types, the matrix elements are different for each subprocess. The leading order (LO) amplitudes can be calculated analytically using the Feynman diagrams at tree level, and are summarized in Table 1. The squared amplitudes are averaged (summed) over the initial (final) color and spin indices, and are expressed in terms of the Mandelstam variables.

Figure 2 shows the matrix elements of the various subprocesses, at LO, as a function of $\cos \theta^{*}$. With the exception of one subprocess $\left(q_{1} \bar{q}_{1} \rightarrow q_{2} \bar{q}_{2}\right)$, there is a characteristic $t$ - channel pole which enhances the two-parton scattering at small angles. Another important feature is the fact that, due to the larger color factor of gluons, the matrix element of the subprocesses with gluons in the initial state lead to larger values.

\subsubsection{Hadronic cross sections}

In a hard scattering process, initiated by colliding hadrons, the experimentally measured cross section can be generally expressed in terms of the parton distribution functions (PDFs) $f(x)$ and the parton-parton scattering cross section $\hat{\sigma}$, summed over all the incoming and outgoing parton types (because the experimentally observed jets cannot distinguish between the parton types):

$$
\sigma=\sum_{i j} \int d x_{1} d x_{2} f_{i}\left(x_{1}, \mu_{F}^{2}\right) f_{j}\left(x_{2}, \mu_{F}^{2}\right) \hat{\sigma}_{i j}\left(\alpha_{s}\left(\mu_{R}^{2}\right), \frac{Q^{2}}{\mu_{F}^{2}}, \frac{Q^{2}}{\mu_{R}^{2}}\right)
$$




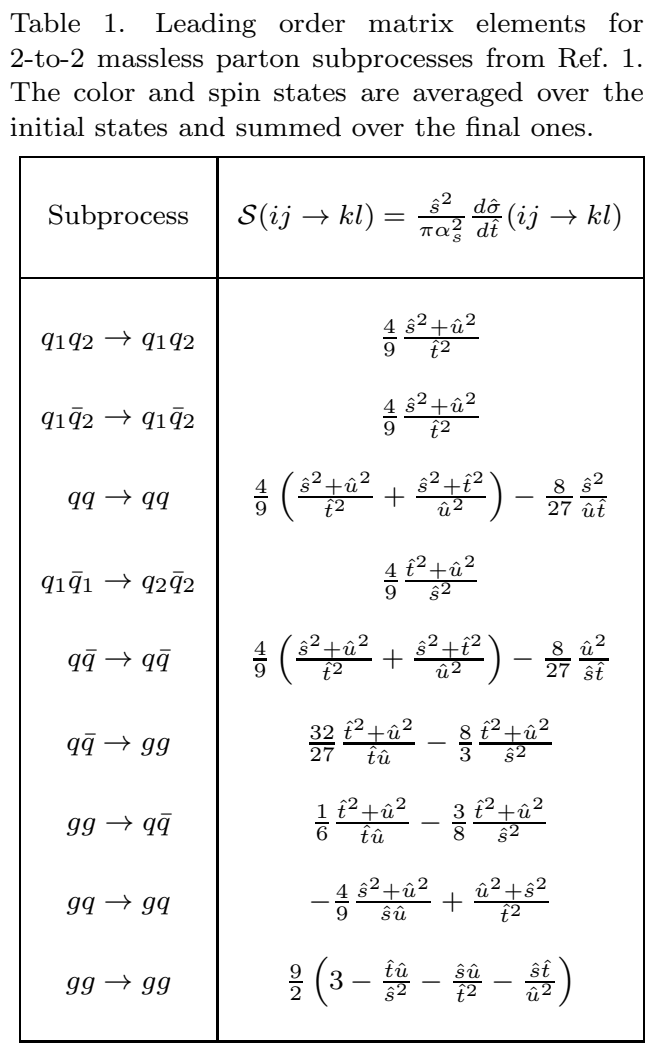

In the equation above, $Q$ is the characteristic hard scale of the interaction (e.g. the dijet invariant mass in a two-to-two parton scattering), $\mu_{F}$ is the factorization scale, which is of the same order as $Q$ and separates the long-distance, non-perturbative interactions from the hard scattering, and $\mu_{R}$ is the renormalization scale. Both the $\mu_{F, R}$ scales are arbitrary parameters of a fixed-order calculation. At all orders of the perturbative expansion, the cross section should be independent of them $\left(\partial \sigma / \partial \mu_{R}=\partial \sigma / \partial \mu_{F}=0\right)$. In all practical calculations of cross sections at a fixed order, it is assumed that $\mu_{R}=\mu_{F}=Q$. It should be noted, that the higher the order of the calculation, the weaker is the dependence on $\mu_{R, F}$.

It is often helpful in hadron collisions to quantify the effect of the parton distribution functions by introducing the parton luminosity factor. This is defined as:

$$
\frac{d L_{i j}}{d \tau}=\int_{0}^{1} \int_{0}^{1} d x_{1} d x_{2} f_{i}\left(x_{1}\right) f_{j}\left(x_{2}\right) \delta\left(x_{1} x_{2}-\tau\right),
$$

where

$$
\tau=x_{1} x_{2}=\frac{\hat{s}}{s} .
$$

In practice, experimental constraints are imposed on the rapidities of the outgoing partons, observed as hadronic jets. It is therefore more convenient to express 


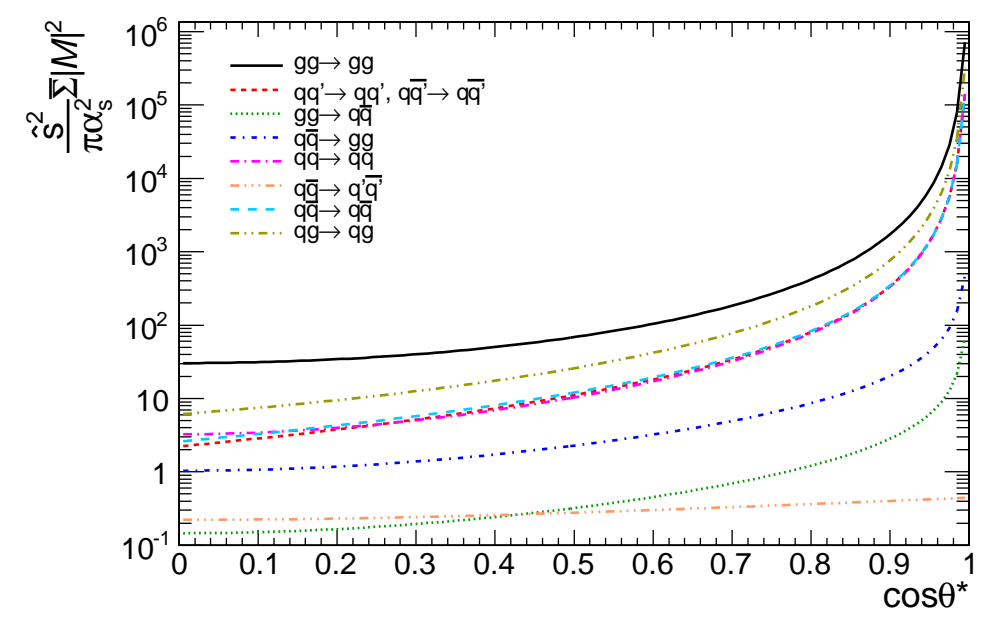

Fig. 2. Leading order matrix elements for two-to-two massless parton scattering, as a function of $\cos \theta^{*}$.

the parton luminosity as a functions of the variables $\tau$ and $\bar{y}$, rather than $x_{1,2}$ : $d x_{1} d x_{2}=\frac{\partial(\tau, \bar{y})}{\partial x_{1}, x_{2}} d \tau d \bar{y}=d \tau d \bar{y}$. The parton luminosity then is:

$$
\frac{d L_{i j}\left(\bar{y}_{\min }, \bar{y}_{\text {max }}\right)}{d \tau}=\int_{\bar{y}_{\text {min }}}^{\bar{y}_{\text {max }}} f_{i}\left(\sqrt{\tau} e^{\bar{y}}\right) f_{j}\left(\sqrt{\tau} e^{-\bar{y}}\right) d \bar{y}
$$

The hadronic cross section of any process, can be expressed generally as a function of the parton luminosity factor and the partonic cross section 2 .

$$
\sigma_{\text {had }}=\sum_{i, j} \int \frac{d \tau}{\tau}\left[\frac{1}{s} \frac{d L_{i j}}{d \tau}\right]\left[\hat{s} \hat{\sigma}_{i j}\right]
$$

In the specific case of a two-to-two scattering, resulting in the production of two jets, the differential cross section can be expressed as a function of the diparton invariant mass and the scattering angle at the center-of-mass frame. To first approximation, this cross section is equal to the observed dijet cross section. The matrix elements presented in table 1 are folded with the parton distribution functions, giving:

$$
\frac{d^{2} \sigma_{h a d}}{d m d \cos \theta^{*}}=\frac{\pi \alpha_{s}^{2}}{m} \sum_{i j}\left[\frac{1}{s} \frac{d L_{i j}}{d \tau}\right]_{\tau=m^{2} / s} \hat{\mathcal{F}}_{i j}\left(\cos \theta^{*}\right)
$$

with

$$
\hat{\mathcal{F}}_{i j}\left(\cos \theta^{*}\right)=\sum_{k l} \mathcal{S}(i j \rightarrow k l) \frac{1}{1+\delta_{k l}} .
$$

In the equations above $m \approx \sqrt{\hat{s}}=\sqrt{\tau s}$ is the dijet invariant mass. 


\subsection{Models of partonic resonances}

\subsubsection{Excited quarks}

In various theoretical models, ordinary quarks can be composite objects 3 , with a characteristic compositeness scale $\Lambda$. As a natural consequence, excited states are expected, called simply excited quarks and denoted by $q^{*}$. Depending on the details of the composite models, the excited quarks can have various values of spin and weak isospin. In the simplest case, they take the value of $1 / 2$. The interaction of excited quarks with the Standard-Model fields is of a "magnetic" type, and the Lagrangian takes the form 4 .

$$
\mathcal{L}=\frac{1}{2 \Lambda} \bar{q}_{R}^{*} \sigma^{\mu \nu}\left(g_{s} f_{s} t_{a} G_{\mu \nu}^{a}+g f \frac{\vec{\tau} \cdot \vec{W}_{\mu \nu}}{2}+g^{\prime} f^{\prime} \frac{Y}{2} B_{\mu \nu}\right) q_{L}+\text { h.c },
$$

where $t_{a}$ and $\vec{\tau}$ are the generators of the color $S U(3)$ and isospin $S U(2), Y$ is the hypercharge, $G_{\mu \nu}^{a}, \vec{W}_{\mu \nu}, B_{\mu \nu}$ are the field tensors, $g_{s}, g, g^{\prime}$ are the gauge couplings, and $f_{s}, f, f^{\prime}$ are dimensionless constants, accounting for possible deviations from the Standard-Model couplings.

In hadron collisions, the production of an excited quark happens through the quark-gluon fusion. Subsequently, the excited quark decays to an ordinary quark and a gauge boson. The dominant decay channel is $q^{*} \rightarrow q g$, leading to a dijet signature. The partial width for the decay of an excited quark with mass $m^{*}$ is given by:

$$
\Gamma\left(q^{*} \rightarrow q g\right)=\frac{1}{3} \alpha_{s} f_{s}^{2} \frac{m^{* 3}}{\Lambda^{2}}
$$

\subsubsection{Randall-Sundrum gravitons}

The gravity model from Randal and Sundrum 56 (RS) was proposed as a solution to the electroweak vs Planck scale hierarchy problem. In this model the hierarchy is generated by an exponential function of the compactification radius of one extra dimension. The metric in the 5 -dimensional space is given by:

$$
d s^{2}=e^{-2 k r_{c} \phi} \eta_{\mu \nu} x^{\mu} x^{\nu}+r_{c}^{2} d \phi^{2}
$$

where $\phi$ is the extra dimension with compactification radius $r_{c}, k$ is a constant of the same order and related to the 5-dimensional Planck scale $M$, and $x^{\mu}$ are the usual space-time dimensions. The reduced effective 4-D Planck scale $\bar{M}_{P l}$ is given by:

$$
\bar{M}_{P l}^{2}=\frac{M^{3}}{k}\left(1-e^{-2 k r_{c} \pi}\right) .
$$

In this model, spin-2 gravitons appear as the Kaluza-Klein (KK) excitations of the gravitational field $h^{\mu \nu}$, whose coupling to the Standard-Model fields is given by 
the interaction Lagrangian 7 :

$$
\mathcal{L}_{I}=-\frac{1}{\Lambda_{\pi}} h^{\mu \nu} T_{\mu \nu}
$$

with $T_{\mu \nu}$ being the energy-momentum tensor of the matter fields. The scale $\Lambda_{\pi}$ and the mass $m_{n}$ of the KK excitations can be expressed as a function of the fundamental constants of the theory:

$$
\Lambda_{\pi}=\bar{M}_{P l} e^{-k r_{c} \pi}, m_{n}=k x_{n} e^{-k r_{c} \pi} .
$$

The coupling constant of the graviton-matter interaction is the inverse of the scale $\Lambda_{\pi}$ :

$$
g=\frac{1}{\Lambda_{\pi}}=x_{n} \frac{\left(k / \bar{M}_{P l}\right)}{m_{n}},
$$

where $x_{n}$ is the n-th root of the the Bessel function of order 1. The phenomenological consequences of the RS-gravitons are essentially determined by their mass, and the ratio $k / \bar{M}_{P l}$. If the fundamental constants of the model satisfy the relation $k r_{c} \sim 12$, then $\Lambda_{\pi} \sim \mathrm{TeV}$, and RS gravitons can be produced in hadron collisions. Through the graviton coupling to the matter fields, RS-gravitons can decay to partons, leading to a dijet signature. The relevant partial widths 8 for the first KK excitation are given by:

$$
\Gamma(G \rightarrow g g)=\frac{x_{1}^{2}}{10 \pi}\left(\frac{k}{\bar{M}_{P l}}\right)^{2} m_{1},
$$

and

$$
\Gamma(G \rightarrow q \bar{q})=\frac{3 x_{1}^{2}}{160 \pi}\left(\frac{k}{\bar{M}_{P l}}\right)^{2} m_{1}
$$

\subsubsection{Axigluons}

In the axigluon models 9 , the symmetry group of the strong sector is expanded to a chiral color group $\mathrm{SU}(3)_{L} \times \mathrm{SU}(3)_{R}$ which, at some energy, breaks to the diagonal SU(3). Under such a symmetry group, the left-handed and right-handed fermions $\psi_{L, R}=\frac{1}{2}\left(1 \mp \gamma_{5}\right) \psi$ transform differently and the transformations are generated by the $T_{L, R}^{a}$ generators. Equivalently, the group can be described by a linear transformation of the generators, divided into vectorial $T_{V}^{a}=T_{L}^{a}+T_{R}^{a}$ and axial $T_{A}^{a}=T_{L}^{a}-T_{R}^{a}$ ones. The associated gauge field to the vectorial generators is identified as the usual color field of QCD, while the gauge field associates to the axial generators is called the axigluon field. While the exact implementation of the chiral color group is model dependent, there are two universal features: the existence of a massive color octet axigluon field (corresponding to the broken symmetry), and the existence of new particles which are needed to cancel the triangular anomalies.

Axigluons can decay to quark-antiquark pairs, which leads to a dijet experimental signature. Note that due to parity conservation, the axigluon cannot decay 
to a gluon-gluon pair (all gluon-axigluon vertices must have an even number of axigluons). The axigluon decay to fermions is described by the Lagrangian:

$$
\mathcal{L}_{A}=-i g_{s}\left(\sum_{i j} \bar{q}_{i} \gamma_{5} \gamma_{\mu} t_{a} q_{j}\right) \mathcal{A}^{a \mu},
$$

where $g_{s}=\sqrt{4 \pi \alpha_{s}}, \mathcal{A}$ is the axigluon field, and $t^{a}$ are the usual color group generators. The width of the axigluon decay can be shown to be 12 .

$$
\Gamma_{A}=\frac{N \alpha_{s} M_{A}}{6}
$$

where $N$ refers to the open decay channels, and $M_{A}$ is the axigluon mass. The latter is a free parameter of the theory, determined by the chiral color symmetry breaking scale and the details of the model.

\subsubsection{Colorons}

Similar to the axial color models, their exist other possibilities to enrich the group structure of the strong sector. Such a model is the flavor-universal coloron 10, where the gauge group is extended to $\mathrm{SU}(3)_{1} \times \mathrm{SU}(3)_{2}$. The corresponding gauge couplings are denoted as $\xi_{1,2}$. Additionally, the model includes a scalar boson $\Phi$, which develops a non-zero vacuum expectation value and breaks spontaneously the symmetry of the two groups. The diagonal subgroup remains unbroken and is identified as the familiar color group of QCD. In the rotated phase of the physical gauge fields, the initial gauge bosons are mixed, forming an octet of massless gluons and an octet of massive colorons. The mass of the colorons is expressed as a function of the fundamental parameters 11 :

$$
M_{C}=\left(\frac{g_{s}}{\sin \theta \cos \theta}\right) f
$$

where $\theta$ is the gauge boson mixing angle with $\cot \theta=\frac{\xi_{2}}{\xi_{1}}$, and $f$ is the vacuum expectation value of the scalar field. The Lagrangian of the interaction between the colorons field $C^{\mu a}$ and the quarks is similar to QCD:

$$
\mathcal{L}=-g_{s} \cot \theta\left(\sum_{i j} \bar{q}_{i} \gamma_{\mu} t_{a} q_{j}\right) C^{\mu a} .
$$

The above interaction predicts the decay of colorons to quarks with kinematically allowed masses. It can be shown that the decay width is:

$$
\Gamma_{C} \approx \frac{N}{6} \alpha_{s} \cot ^{2} \theta M_{C}
$$

where $N$ is the number of quark flavors with mass less than $M_{C} / 2$. 


\subsubsection{Color octet scalars}

In various theoretical models, bosonic states can arise from gluon-gluon fusion. The color octet scalar model $\left(S_{8}\right)$ is one example of exotic color resonances 13 . The coupling of the color octet scalar field with gluons is expressed with the Lagrangian:

$$
\mathcal{L}=g_{s} \frac{\kappa}{\Lambda} d^{a b c} S_{8}^{a} G_{\mu \nu}^{b} G^{c, \mu \nu},
$$

where $g_{s}$ is the strong coupling constant, $\kappa$ is the scalar coupling, $\Lambda$ is the characteristic scale of the interaction, $d^{a b c}$ are structure constants of the $\mathrm{SU}(3)$ group defined by the relation $\left\{t^{a}, t^{b}\right\}=\frac{1}{3} \delta^{a b}+d^{a b c} t^{c}$, and $S_{8}, G_{\mu \nu}$ are the color octet scalar field, and the gluon field tensor, respectively. The width of the color octet scalar resonance is given by:

$$
\Gamma=\frac{5}{6} \alpha_{s} \kappa^{2} \frac{M^{3}}{\Lambda^{2}}
$$

\subsection{6. $Z^{\prime}$ and $W^{\prime}$}

New gauge bosons arise in models where the symmetry $\mathrm{SU}(2)_{L} \otimes \mathrm{U}(1)_{Y}$ of the electroweak Standard-Model sector is enlarged. Common features in these models are the new gauge coupling constants, which are of the same order as the $\mathrm{SU}(2)_{L}$ coupling of the Standard Model, and the existence of new gauge bosons, namely $W^{\prime}$ and $Z^{\prime}$. Under the assumption that the new gauge bosons couple to ordinary quarks and leptons similar to their Standard-Model counterparts, the cross sections of these particles are calculated by scaling the the corresponding Standard-Model cross section. In particular, the Fermi constant $G_{F}$ becomes 14 :

$$
G_{F}^{\prime}=G_{F}\left(\frac{M}{M^{\prime}}\right)^{2}
$$

where $M$ and $M^{\prime}$ are the masses of $W$ or $Z$, and $W^{\prime}$ or $Z^{\prime}$, respectively.

\subsubsection{String resonances}

According to the string theory, particles are created by vibrations of relativistic strings, with mass $M_{s}$, and they populate Regge trajectories, which relate their spins and masses. In principle, the mass of the fundamental strings is of the order of the Planck scale. However, in some theories with large extra dimensions, it is plausible that $M_{s}$ lies in the $T e V$ scale. In this case, Regge excited states of quarks and gluons occur in hadron collisions. If the string coupling is small, the basic properties of the Regge excitations (production cross section and width) are model independent (the details of the compactification are irrelevant). This statement is true for parton scattering involving gluons, but only approximately true in the four-quark scattering.

The effect of the Regge excitations can be quantified $15[16$ through the presence of a common form factor in the two-to-two parton scattering amplitudes, which is 
called the Veneziano form factor and is written in terms of the $\Gamma$-function:

$$
V(\hat{s}, \hat{t}, \hat{u})=\frac{\Gamma\left(1-\hat{s} / M_{s}^{2}\right) \Gamma\left(1-\hat{u} / M_{s}^{2}\right)}{\Gamma\left(1-\hat{t} / M_{s}^{2}\right)}
$$

where $\hat{s}, \hat{t}, \hat{u}$ are the usual Mandelstam variables. The physical content of the Veneziano form factor is revealed by an expansion in terms of s-channel poles. Each such pole represents a virtual Regge resonance, with mass $\sqrt{n} M_{s}$. For the purpose of resonances in the dijet spectrum, only the first-level $(n=1)$ excitation is relevant, while the string mass $M_{s}$ is the only free parameter. The exact values of the cross sections depend also on the color factors and spin values of the excited states.

\subsection{8. $E_{6}$ Diquarks}

In the context of superstring theory in 10 dimensions, anomaly cancellation requires that the gauge group is $E_{8} \times E_{8}$. Certain models for the compactification of the additional 6 dimensions, predict that the grand unification symmetry group is $E_{6}$ 17. The $E_{6}$ models $\frac{18}{18}$ contain color-triplet scalar diquarks, $D$ and $D^{c}$ with charges $-\frac{1}{3}$ and $\frac{1}{3}$ respectively, which couple to the light quarks $u, d$.

The interaction Lagrangian between the $E 6$ diquarks and the light quarks is given by 19 :

$$
\mathcal{L}=\lambda \epsilon_{i j k} \bar{u}^{c i} \frac{1}{2}\left(1-\gamma_{5}\right) d^{j} D^{k}+\frac{\lambda_{c}}{2} \epsilon_{i j k} \bar{u}^{i} \frac{1}{2}\left(1+\gamma_{5}\right) d^{c j} D^{c k}+h . c,
$$

where $i, j, k$ are color indices, and $\lambda, \lambda_{c}$ are parameters of the hyper-potential of the general $E_{6}$ model. The squared amplitudes for the diquark decays to light quarks are given by 20 :

$$
|\mathcal{M}(D \rightarrow \bar{u} \bar{d})|^{2}=24 \lambda^{2} m_{D}^{2}, \quad\left|\mathcal{M}\left(D^{c} \rightarrow u d\right)\right|^{2}=6 \lambda_{c}^{2} m_{D_{c}}^{2} .
$$

The corresponding widths are:

$$
\Gamma_{D}=\alpha_{\lambda} M_{D}, \quad \Gamma_{D^{c}}=\frac{1}{4} \alpha_{\lambda_{c}} M_{D^{c}}
$$

with $\alpha_{\lambda}=\lambda^{2} / 4 \pi, \alpha_{\lambda_{c}}=\lambda_{c}^{2} / 4 \pi$.

\subsubsection{Color octet technirho}

Technicolor models predict a rich spectrum of technirho vector mesons $\left(\rho_{T}\right)$ that decay predominantly into technipion $\left(\pi_{T}\right)$ states. In most of the models, $\rho_{T}$ is either heavy, leading to small production cross section, or its decay width to $\pi_{T}$ is very large, leading to broad peak structures on the dijet mass spectrum. However, in the context of the "walking technicolor" models 21 , the technifermion chiral condensate acquires large values, resulting in $\pi_{T}$ with large mass, such that the $\rho_{T} \rightarrow \pi_{T} \pi_{T}$ decay is kinematically suppressed. Furthermore, if the technirho is a color multiplet, its production cross section in hadron collisions through the $q \bar{q}, g g \rightarrow \rho_{T}$ 
subprocesses is enhanced. In retrospect, giving the kinematically suppressed decay to technipions, $\rho_{T}$ decays almost $100 \%$ to partons, appearing as a narrow resonance in the dijet mass spectrum.

It should be noted that the phenomenology of the walking technicolor models can be fairly complex 22 , with one reason being the fact that the $\rho_{T}$ states are mixed with ordinary gluons. Because of the matrix form of the corresponding propagator, the predicted resonance on the dijet mass spectrum appears higher than the pole mass. Also, the resonance cross section cannot be calculated independently of QCD, to which it is coupled. For the experimental searches, a simple benchmark model is considered 23, which predicts a single "bump" on the dijet mass spectrum. The corresponding parameters are listed below:

- standard topcolor-assisted-technicolor (TC2) couplings

- degenerate technirhos: $M\left(\rho_{11}\right)=M\left(\rho_{12}\right)=M\left(\rho_{21}\right)=M\left(\rho_{22}\right)=M(\rho)$ (the pole mass)

- mixing among the technirhos: $M_{8}^{\prime}=0$, which reduces the mass shift from the pole mass,

- octet technipion mass: $M\left(\pi_{22}^{8}\right)=\frac{5 M(\rho)}{6}$, which suppresses the decay $\rho_{T} \rightarrow$ $\pi_{T} \pi_{T}$

- singlet technipion mass: $M\left(\pi_{22}^{1}\right)=M\left(\pi_{22}^{1}\right) / 2$,

- coloron mass: $M(V 8) \rightarrow \infty$, so that the coloron does not affect the cross section,

- mass parameter $M_{8}=\frac{5 M(\rho)}{6}$, which keeps the branching fraction of the process $\rho_{T} \rightarrow g \pi_{T}$ small and leads to narrow resonance

\subsubsection{Benchmark Models}

The parton-parton resonance models presented in the previous sections involve limited number of free parameters each. The experimental searches traditionally consider benchmark models, with certain parameter assumptions, which are then used to set limits on the masses of the corresponding resonances. Below is a summary of the benchmark models:

- Axigluons: the number of quark flavors to which the axigluon can decay is set to $N=6$, corresponding to the known quarks.

- Colorons: the number of quark flavors to which the coloron can decay is set to $N=6$, and the gauge boson mixing angle is set to $\cot \theta=1$.

- Excited quarks: standard model couplings are assumed $\left(f_{s}=f=f^{\prime}=1\right)$ and the compositeness scale is set equal to the excited quark mass $\Lambda=M^{*}$.

- $R S$ graviton: the ratio $k / \bar{M}_{P l}$ is set to $k / \bar{M}_{P l}=1$.

- $W^{\prime}, Z^{\prime}$ : standard model couplings are assumed.

- $E_{6}$ diquark: electromagnetic coupling constants are assumed $\alpha_{\lambda}=\alpha_{\lambda_{c}}=$ $\alpha_{e}$. 
- Color octet scalars: the gauge coupling is set equal to the QCD coupling $(\kappa=1)$, and the characteristic scale of the interaction is set equal to the resonance mass $\Lambda=M$.

Table2 2 summarizes the basic properties of the resonances discussed in this review. In particular, the decay widths are approximate values for the benchmark models, since they also depend on the running of $\alpha_{s}$ which should be evaluated at a scale equal to the resonance mass. For string resonances the decay width varies significantly, depending on the spin and color quantum numbers of the resonances.

Table 2. Summary of resonances considered in this review.

\begin{tabular}{|c|c|c|c|c|c|}
\hline Resonance & Symbol & $J^{P}$ & $\begin{array}{c}\text { Color } \\
\text { Multiplet }\end{array}$ & $\Gamma /\left(2 m_{R}\right)$ & $\begin{array}{c}\text { Decay } \\
\text { Channel }\end{array}$ \\
\hline excited quark & $q^{*}$ & $1 / 2^{+}$ & triplet & 0.02 & $q g$ \\
axigluon & $A$ & $1^{+}$ & octet & 0.05 & $q \bar{q}$ \\
coloron & $C$ & $1^{-}$ & octet & 0.05 & $q \bar{q}$ \\
RS graviton & $G$ & $2^{-}$ & singlet & 0.01 & $q \bar{q}, g g$ \\
$E_{6}$ diquark & $D_{6}$ & $0^{+}$ & triplet & 0.004 & $u d$ \\
color octet scalar & $S_{8}$ & $0^{+}$ & octet & 0.04 & $g g$ \\
color octet technirho & $\rho_{T}$ & $1^{-}$ & octet & 0.01 & $q \bar{q}, g g$ \\
heavy W & $W^{\prime}$ & $1^{-}$ & singlet & 0.01 & $q \bar{q} 2$ \\
heavy Z & $Z^{\prime}$ & $1^{-}$ & singlet & 0.01 & $q \bar{q}$ \\
string & $S$ & various & various & $0.003-0.037$ & $q \bar{q}, q g, g g$ \\
\hline
\end{tabular}

\subsubsection{Model Calculations}

The exact LO calculations of the cross sections and the decay widths of the various resonances involve all the relevant Feynman diagrams associated with each Lagrangian. In practice, the experimental searches presented in this review are focused on narrow resonances, which appear as "bumps" on a steeply falling dijet mass spectrum. In all the cases, it is the $s$ - channel decay mode of the resonances which produces a "bump".

The cross section of a resonance decaying through the $s-$ channel is given by the Breit-Wigner expression:

$$
\hat{\sigma}(m)(1+2 \rightarrow R \rightarrow 3+4)=16 \pi \mathcal{N} \times \frac{\Gamma(1+2 \rightarrow R) \times \Gamma(R \rightarrow 3+4)}{\left(m^{2}-m_{R}^{2}\right)^{2}+m_{R}^{2} \Gamma_{R}^{2}},
$$

where $m_{R}$ and $\Gamma_{R}$ are the mass and the total width of the resonance, respectively, $\Gamma(1+2 \rightarrow R)$ and $\Gamma(R \rightarrow 3+4)$ are the partial widths for the creation and the decay of the resonance to the specific final state. The spin and color multiplicity factor $\mathcal{N}$ is

$$
\mathcal{N}=\frac{N_{S_{R}}}{N_{S_{1}} N_{S_{2}}} \frac{C_{R}}{C_{1} C_{2}}
$$


where $N_{S_{R}}, N_{S_{1,2}}$ are the spin multiplicities of the resonance and the initial state particles, while $C_{R}$ and $C_{1,2}$ are the corresponding color factors. The cross section above arises after integrating over $\cos \theta^{*}$. The angular dependence of the cross section, in the $s$ - channel decay mode, is determined by the spin of the resonance and the spin of the final state particles. It should be noted, that in all resonance cases decaying to two partons, the angular dependence is expressed as a polynomial of $\cos \theta^{*}$, as opposed to the dominant QCD background, which exhibits a $t-$ channel pole at $\cos \theta^{*} \rightarrow 1$. More specifically, the angular distributions of the various resonances, in the $s$ - channel decay mode, are listed below:

- $E_{6}$ diquark, color octet scalars: $F\left(\cos \theta^{*}\right)=$ const.

- excited quark: $F\left(\cos \theta^{*}\right) \sim 1+\cos \theta^{*}$, which becomes $F\left(\left|\cos \theta^{*}\right|\right)=$ const. (odd in $\cos \theta^{*}$ ).

- axigluon, coloron, $W^{\prime}, Z^{\prime}: F\left(\cos \theta^{*}\right) \sim 1+\cos ^{2} \theta^{*}$.

- RS gravitons: $F(g g \rightarrow G \rightarrow q \bar{q})=F(q \bar{q} \rightarrow G \rightarrow g g) \sim 1-\cos ^{4} \theta^{*}$, $F(g g \rightarrow G \rightarrow g g) \sim 1+6 \cos ^{2} \theta^{*}+\cos ^{4} \theta^{*}$, and $F(q \bar{q} \rightarrow G \rightarrow q \bar{q}) \sim$ $1-3 \cos ^{2} \theta^{*}+4 \cos ^{4} \theta^{*}$.

where $F\left(\cos \theta^{*}\right) \equiv d \hat{\sigma} / d \cos \theta^{*}$.

In practice, experimental searches impose kinematic constraints on the scattering angle $\theta^{*}$, such that the QCD background is suppressed. In this case, the BreitWigner partonic cross section is written as:

$$
\hat{\sigma}(m)=\frac{16 \pi \times \mathcal{N} \times \mathcal{A}_{\cos \theta^{*}} \times B R \times \Gamma_{R}^{2}}{\left(m^{2}-m_{R}^{2}\right)^{2}+m_{R}^{2} \Gamma_{R}^{2}},
$$

where $B R$ is the branching fraction of the subprocess, and $\mathcal{A}_{\cos \theta^{*}}$ is the acceptance after the $\cos \theta^{*}$ cut. If the resonance is sufficiently narrow $\left(\Gamma_{R}<<m_{R}\right)$, the narrowwidth approximation holds:

$$
\frac{1}{\left(m^{2}-m_{R}^{2}\right)^{2}+m_{R}^{2} \Gamma_{R}^{2}} \approx \frac{\pi}{m_{R} \Gamma_{R}} \delta\left(m^{2}-m_{R}^{2}\right) .
$$

Finally, the hadronic cross section in the narrow-width approximation is derived:

$$
\sigma_{\text {had }}\left(m_{R}\right)=16 \pi^{2} \times \mathcal{N} \times \mathcal{A}_{\cos \theta^{*}} \times B R \times\left[\frac{1}{s} \frac{d L\left(\bar{y}_{\text {min }}, \bar{y}_{\text {max }}\right)}{d \tau}\right]_{\tau=m_{R}^{2} / s} \times \frac{\Gamma_{R}}{m_{R}},
$$

where the parton luminosity $\frac{d L}{d \tau}$ is calculated at $\tau=m_{R}^{2} / s$, and constrained in the kinematic range $\left[\bar{y}_{\min }, \bar{y}_{\max }\right]$.

\section{Experiment}

In this review paper we consider all searches that used the dijet mass or $p_{T}$ spectra to search for dijet resonances. The searches considered are listed in table 3 in chronological order, along with a summary of the energy and luminosity of the dataset, and the techniques of the search. The searches by the UA1 and UA2 experiments used data from the proton anti-proton collisions at the CERN $S \bar{p} p S$ collider 
at a center-of-mass energy of $0.63 \mathrm{TeV}$. The searches by the CDF and D0 experiments used data from proton anti-proton collisions at the Fermilab Tevatron at a center-of-mass energy of 1.8 and $1.96 \mathrm{TeV}$. The searches by the ATLAS and CMS experiments used data from proton proton collisions at the CERN Large Hadron Collider at a center-of-mass energy of $7 \mathrm{TeV}$. The search techniques listed in table 3 are discussed in the following sections.

Table 3. Searches for dijet resonances at hadron colliders. For each search we list the experiment, year of data publication, center-of-mass energy, integrated luminosity, techniques used to define the resonance shape and the background, dijet mass range of the data, the cut applied on the center of mass scattering angle, and the primary reference for the search.

\begin{tabular}{|c|c|c|c|c|c|c|c|c|}
\hline Expt. & Yr. & $\begin{array}{c}\sqrt{s} \\
(\mathrm{TeV})\end{array}$ & $\begin{array}{l}\int L d t \\
\left(p b^{-1}\right)\end{array}$ & $\begin{array}{l}\text { Resonance } \\
\text { Shape }\end{array}$ & $\begin{array}{c}\text { Background } \\
\text { Shape }\end{array}$ & $\begin{array}{l}m_{J J} \\
(\mathrm{TeV})\end{array}$ & $\begin{array}{c}\cos \theta^{*} \\
\text { Cut }\end{array}$ & $\begin{array}{c}\text { Ref. } \\
\#\end{array}$ \\
\hline UA1 & 86 & 0.63 & 0.26 & BW $\oplus$ Gaussian & LO QCD & $.07-0.3$ & - & 12 \\
\hline UA1 & 88 & 0.63 & 0.49 & $\mathrm{BW} \oplus$ Gaussian & LO QCD & $.11-0.3$ & bins & 25 \\
\hline $\mathrm{CDF}$ & 90 & 1.8 & 0.026 & BW $\oplus$ Gaussian & LO QCD & $.06-0.5$ & - & 26 \\
\hline UA2 & 90 & 0.63 & 4.7 & Gaussian & Fit Func. & $.05-0.3$ & - & 27 \\
\hline $\mathrm{CDF}$ & 93 & 1.8 & 4.2 & $\mathrm{BW} \oplus$ Resolution & LO QCD & $.14-1.0$ & - & 28 \\
\hline UA2 & 93 & 0.63 & 11 & Gaussian & Fit Func. & $.05-0.3$ & .60 & 29 \\
\hline $\mathrm{CDF}$ & 95 & 1.8 & 19 & Рүтніа $\oplus$ Sim. & Fit Func. & $.15-0.9$ & .67 & 30 \\
\hline $\mathrm{CDF}$ & 97 & 1.8 & 106 & Рүтніа $\oplus$ Sim. & Fit Func. & $.18-1.0$ & .67 & 31 \\
\hline D0 & 04 & 1.8 & 109 & РутніА $\oplus$ Sim. & NLO QCD & $.18-1.2$ & .67 & 32 \\
\hline $\mathrm{CDF}$ & 09 & 1.96 & 1130 & Рүтніа $\oplus$ Sim. & Fit Func. & $.18-1.3$ & - & 33 \\
\hline ATLAS & 10 & 7 & 0.32 & Pyтніа $\oplus$ Sim. & Fit Func. & $.20-1.7$ & .57 & 34 \\
\hline CMS & 10 & 7 & 2.9 & РүтніА $\oplus$ Sim. & Fit Func. & $.22-2.1$ & .57 & 35 \\
\hline ATLAS & $11 \mathrm{w}$ & 7 & 36 & РүтніА $\oplus$ Sim. & Fit Func. & $.50-2.8$ & .57 & 36 \\
\hline CMS & 11 & 7 & 1000 & PyтніA $\oplus$ Sim. & Fit Func. & $.84-3.7$ & .57 & 37 \\
\hline ATLAS & $11 \mathrm{~s}$ & 7 & 1000 & РүтніА $\oplus$ Sim. & Fit Func. & $.72-4.1$ & .54 & 38 \\
\hline
\end{tabular}

\subsection{Resonance Shapes}

Narrow resonances are those whose observed width is dominated by experimental resolution, where the natural width of the resonance is small in comparison. Searches for narrow resonances are prevalent in high energy physics, because they in principle only require the use of one shape for the signal, dominated by the experimental resolution. This is an approximation, as we will see explicitly in this section.

Searches for dijet resonances have modeled the resonance line shape using either an analytic or a Monte Carlo technique, as shown in Table 3 .

The analytic technique used by the older searches started with a natural shape for the resonance which was then smeared with the experimental resolution function. That natural shape was either a Breit-Wigner (BW) for wide resonances, where the natural width could not be ignored, or a delta-function for narrow resonances. The principle of the technique is the idea that the resolution can be understood separately from the natural line shape, and the two can then be convolved. In practice things were often done this way in older searches because of limitations in 
the MC modeling or the detector simulation. The search 12 using 1986 data from UA1 ${ }^{24}$ considered the entire spectrum, with QCD and resonances determined analytically and summed together, and then smeared with the experimental resolution, $\sigma\left(p_{T}\right) / p_{T}=0.53 / \sqrt{p_{T}}+0.05$. The UA1 search in $1988^{25}$ smeared a BW shape in a toy $\mathrm{MC}$ with the Gaussian experimental dijet mass resolution, $\sigma / m=0.11$, and checked the process with the IsAJET MC 39 and a full UA1 simulation. The UA2 search in 199027 , and again in 19932 , considered only narrow resonances and used the Gaussian experimental resolution for the resonance shapes. The resolution varied from $10.7 \%$ at $80 \mathrm{GeV}$ to $8.4 \%$ at $300 \mathrm{GeV}$ for $W^{\prime} \rightarrow q \bar{q}$ resonances, and UA2 noted that the resolution was worse for a $g g$ resonance, $13.2 \%$ at $80 \mathrm{GeV} 27$. The CDF searches in 199026 and 1993228 coherently summed an axigluon resonance shape to the QCD background and then smeared with the experimental dijet mass resolution. The resolution was $\sigma / m=0.68 / \sqrt{m}+0.065$ in 1990, and in 1993 it was parameterized in a more complex way and also included the effects of QCD radiation from HeRwIG 40 . The 1990 search was for narrower axigluons, $N=5$ and 10, and the 1993 search was for wider axigluons, $N=10$ and 20. The 1993 paper also considered a more generic search for three widths, one narrow $(\Gamma=0.02 M)$ and two wide $(\Gamma=0.1 M$ and $0.2 M)$.
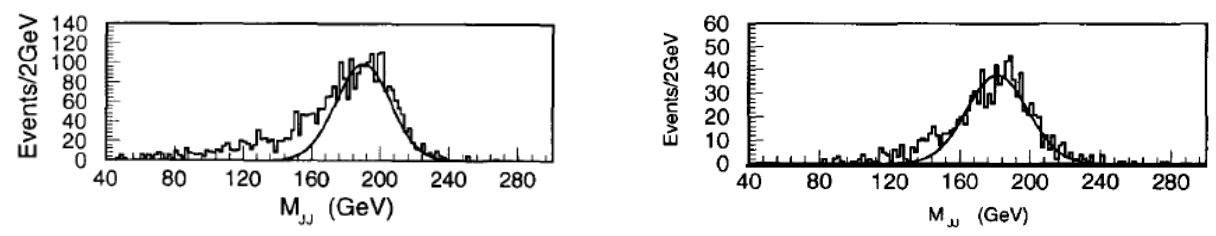

Fig. 3. Resonance shapes from UA2 in 1993. (left) $Z^{\prime}$ and (right) excited quark simulations at a mass of $200 \mathrm{GeV}$ with Gaussian fits overlaid. Reprinted from Ref. 29] Copyright 1993, with permission from Elsevier.

A nice early illustration of narrow resonance shapes was provided by UA2 in 199329 and is reproduced in Fig. 3. The Gaussian shapes used to model the signal represent reasonably well the central core of the simulated distribution using PүтнiA 41, but there is also a long tail at low mass due to final state radiation. UA2 accounted for the tail using a signal efficiency, and stated that "this tail is re-absorbed in the parameterization of the QCD background". The tail at low mass is generally negligible compared to the QCD background, so a Gaussian was an adequate approximation. Nevertheless, all subsequent searches used the full resonance shape including the radiative tail to fit for resonances and set limits.

The MC technique used by the more recent searches modeled the resonance line shape using PYTHIA with either a fast or full detector simulation. The CDF searches in $1995 \stackrel{30}{30}$ and $1997 \stackrel{31}{\longrightarrow}$ used an excited quark decaying to $q g$ to model the resonance shape for all dijet resonances. Uncertainties on the amount of final 

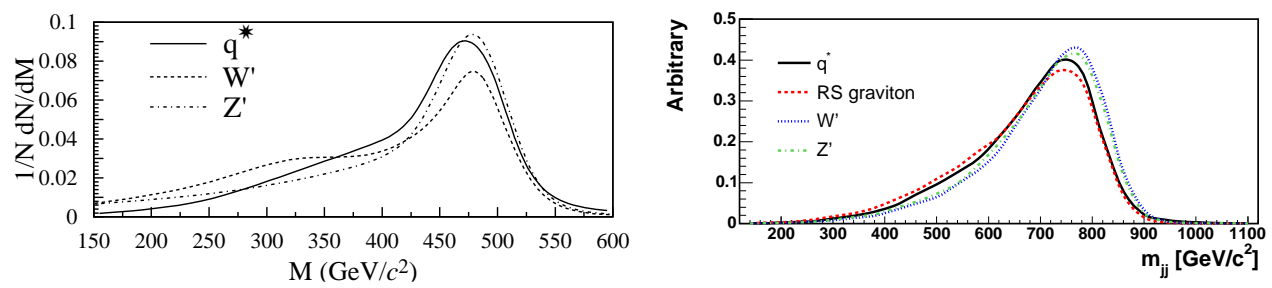

Fig. 4. Resonance shapes from D0 in 2004 and CDF in 2009. (left) D0 simulation of three models of a $500 \mathrm{GeV}$ resonance from Ref. 32, Copyright 2004, and (right) CDF simulation of four models of an $800 \mathrm{GeV}$ resonance from Ref. 33 . Copyright 2009 by the American Physical Society.

state radiation were included by changing the amount of the "radiation tail" at low mass by $\pm 50 \%$. The Gaussian core of the dijet mass resolution was reported as approximately $10 \%$ at $\mathrm{CDF}$ 30|31/33, but the resolution did depend on dijet mass. The D0 search in $2004^{32}$, and the CDF search in 2009 33 , used independent shapes for different models of narrow resonances available in PүтніA, as shown in Fig. 4. The shapes varied primarily due to the different amounts of final state radiation, with the low mass tail and the width usually increasing with the number of gluons in the final state.

The CMS search in 2010 35 introduced independent narrow resonance shapes for each generic type of final state: quark-quark, quark-gluon, and gluon-gluon shown in Fig. 15. They reported in Ref. 35 that for resonance masses between 0.5 and 2.5 $\mathrm{TeV}$ the Gaussian resolution of the core of the distribution "varies from $8 \%$ to 5\% for $q q, 10 \%$ to $6 \%$ for $q g$, and $16 \%$ to $10 \%$ for $g g$ ". These were estimated by fitting mainly the peak and high-mass edge to a Gaussian 54. To reduce the radiation tail the CMS search in 201137 introduced wide-jets with an effective radius of $\Delta R=1.1$. Wide-jets also improved the Gaussian component of the resolution which in 2011 was $\sigma / M=1.31 / \sqrt{M}+0.018$ for $q q, \sigma / M=1.56 / \sqrt{M}+0.027$ for $q q$, and $\sigma / M=2.09 / \sqrt{M}+0.015$ for $g g$ resonances, with resonance mass $M$ given in $\mathrm{GeV}$, and between 500 and $3500 \mathrm{GeV} 42$. The improvement is largest for gluon-gluon resonances, as shown in Fig. 5 where the shape using wide-jets is compared with the shape using narrower jets from the anti- $k_{T}$ algorithm 43 with a distance parameter $R=0.7$.

The ATLAS search in $2010 \stackrel{34}{34}$ considered the explicit dijet resonance shape of the excited quark model from PyтHIA, "including all possible SM final states, which were dominantly $q g$ but also $q W, q Z$, and $q \gamma$ ". The reported Gaussian resolution for an excited quark ranged from $11 \%$ to $7 \%$ for $q^{*}$ masses between 0.3 and $1.7 \mathrm{TeV} \underline{34}$, and the reported detector level resolution $\sigma_{m_{j j}} / m_{j j}$ was " $5 \%$ at $1 \mathrm{TeV}, 4.5 \%$ at $2 \mathrm{TeV}$, and asymptotically approached $4 \%$ at masses of $5 \mathrm{TeV}$ and above" 38 . In 201136 ATLAS reused the $q^{*}$ shapes to set limits on axigluons, after noting that "the axigluon and $q^{*}$ signal templates result in very similar limits". In summer 201138 ATLAS introduced a shape for $g g$ resonances. The ATLAS searches in 
$2013_{36 / 38}^{36}$ also considered generic limits for Gaussian shaped resonances of varying width, not just narrow resonances.

The shapes of narrow resonances presented in the literature are all well described by a Gaussian core and a radiation tail, but the different resonance shapes in a single experiment also demonstrates that the concept of narrow resonances is often approximate.
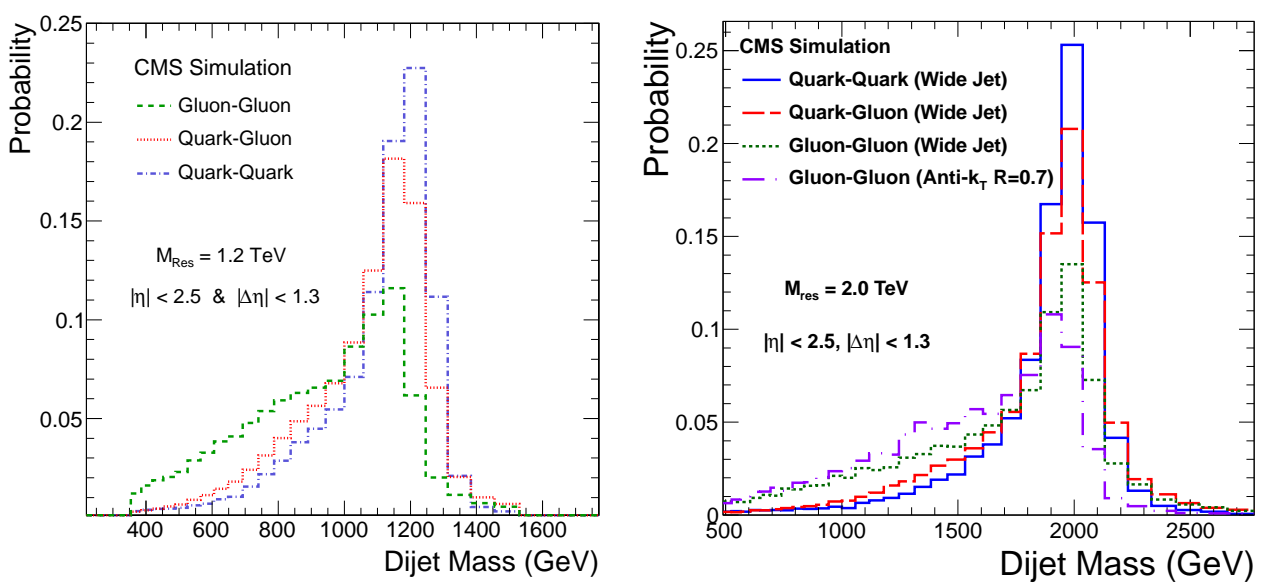

Fig. 5. Resonance shapes from CMS. (left) Simulations of $1.2 \mathrm{TeV}$ resonances shown for the different possible final state partons, from Ref. 35. Copyright 2010 by the American Physical Society. (right) Simulation of $2 \mathrm{TeV}$ resonances reconstructed with two different types of jets, from Ref. 37. Copyright 2011, with permission from Elsevier.

In addition to radiation, another parton-level physical mechanism produces a significant tail at low mass, further perturbing the narrow resonance search technique: the parton luminosities at low mass are larger than at the resonance pole mass, and significantly lift the natural Breit-Wigner tail of the resonances, in the cases where the PDFs are falling off rapidly with increasing dijet mass. This frequently happens at resonance masses approaching the kinematic bound for both sea quarks and gluons, and the size of the effect increases with the resonance intrinsic width, even for resonances normally considered narrow. The extreme end of this tail due to the PDFs is sometimes suppressed in the searches by requiring the partons to be have mass close to the pole mass, within a few standard deviations on the dijet mass resolution. This is generally a reasonable solution for the shapes, as the QCD background overwhelms the signal at low dijet mass. However, the way that this tail from PDFs is handled can significantly affect the total resonance cross section quoted for specific models, as we discuss in Appendix A.

The narrow resonance search technique, where a single resonance shape dominated by experimental resolution is used to model the effect of all narrow resonances, 
is only strictly applicable when the half-width of the resonance, $\Gamma / 2$ is significantly less than the experimental Gaussian resolution $\sigma$. The model half-widths are listed in table 2, and vary from about $0.4 \%$ to as large as $\alpha_{s} / 2$ for axigluons and colorons. For the searches at UA1, UA2, CDF, and D0, $\alpha_{s} / 2$ is about a factor of 2 less than the dijet mass resolution of roughly $10 \%$, and the half-widths of the other models are significantly less than $10 \%$, so the narrow resonance technique should be applicable. However, the searches at CMS and ATLAS are now looking for very massive resonances, producing very energetic jets measured with better resolution. For an axigluon or coloron of mass $3 \mathrm{TeV}$ the half-width is $\alpha_{s}(3 \mathrm{TeV}) / 2=3.9 \%$. For a resonance mass of $3 \mathrm{TeV}$, the Gaussian dijet mass resolution at CMS in the $q q$ channel in 2011 is $4.2 \%$, and at ATLAS at $3 \mathrm{TeV}$ the dijet mass resolution quoted above is roughly the same. In addition, both CMS and ATLAS have also included a long tail to low mass due to radiation, which is a part of the modification of the natural line width into an observed line width, so the experimental resolution is somewhat worse than $4.2 \%$. Nevertheless, the natural half-width of $3.9 \%$ is comparable to the experimental resolution, and so the widest resonances ATLAS and CMS have considered, like axigluons and colorons, are beginning to push the boundaries of the narrow resonance classification.

\subsection{Dijet Data and QCD Background}

The heart of the search for dijet resonances is the measurement of the dijet mass distribution and the estimation of the background. Unlike many other searches in high energy physics, the search for dijet resonances is completely dominated by a single background. The observed dijet mass distribution comes from the dominant process in hadronic collisions: $2 \rightarrow 2$ scattering of partons predicted by perturbative QCD.

\subsubsection{Angular Requirement}

The event selection requirements of each search can only be understood by examining the dijet production in QCD. Most experiments chose a fiducial region in the experiment to measure dijet production, limiting the pseudorapidity, $\eta$, of each jet to a central region. In addition, as shown in table 3. many of the searches place a cut on the center of momentum frame scattering angle $\left|\cos \theta^{*}\right|$ b Both of these selections, but the $\left|\cos \theta^{*}\right|$ one in particular, are designed to enhance the dijet resonance signal and suppress the QCD background. This is because QCD production of dijets at high mass is dominated by $t$-channel production, with an angular distribution that is approximately Rutherford scattering (Section 2.1.4):

$$
\frac{d N}{d \cos \theta^{*}} \sim \frac{1}{\hat{t}^{2}} \sim \frac{1}{\left(1-\cos \theta^{*}\right)^{2}}
$$

\footnotetext{
${ }^{\mathrm{b}}$ For some experiments we have translated their cut on dijet $\Delta \eta$ or $\Delta y$ to the equivalent cut on $\cos \theta^{*} \approx \tanh (\Delta \eta / 2)$, which follows from Eq. 5 and $\Delta \eta \approx \Delta y$.
} 
peaking strongly at $\cos \theta^{*}=1$. In practice, only $\left|\cos \theta^{*}\right|$ is measured, because the partons in the final state emerge as jets and are experimentally indistinguishable. In the $s$ - channel mode, dijet resonances lead to angular distributions that are much flatter in $\cos \theta^{*}$ than QCD, with the exact angular distribution depending on the spin of the resonance and the final state partons (Section 2.2.11). The optimal $\left|\cos \theta^{*}\right|$ cut is determined by maximizing the integrated signal, $\mathrm{S}$, over the square root of the integrated background, $\sqrt{B}$, often by using a Monte Carlo. However, one can estimate the optimal $\cos \theta^{*} \operatorname{cut}$, using equation 45 for the background and a signal flat in $\left|\cos \theta^{*}\right|$, resulting in the optimal cut $\left|\cos \theta_{M A X}^{*}\right|=1 / \sqrt{3}=0.58$. Table 3 shows that all experiments which employed a cut on $\left|\cos \theta^{*}\right|$ used a similar value in order to optimize their searches for dijet resonances.

\subsubsection{Background Models}

Searches for dijet resonances have chosen to model the background with either a QCD calculation or with a background parameterization, as shown in table 3. No search has used a Monte Carlo simulation to model the QCD background, even if they used a Monte Carlo to model the resonance signal. This is because Monte Carlo may not model the spectrum shape accurately enough. QCD calculations are also challenged by high statistics jet data. In the cases where leading order (LO) QCD was used to model the spectrum shape, the normalization of LO QCD was always adjusted to obtain agreement with the data. Despite the considerable progress in next-to-leading order (NLO) QCD calculations over the last 20 years, only one experiment has successfully used NLO QCD to model the dijet background 32 , and remarkably was able to use the absolute normalization of the calculation. Even when normalization and shape agree between QCD and the data, there are still considerable theoretical uncertainties (PDFs, renormalization scale, non-perturbative effects, ...) and experimental uncertainties (jet energy scale, resolution smearing, ...) which can produce significant differences between data and QCD that can potentially mimic a resonance signal. For these reasons, the majority of searches simply used a fit function to parametrize the QCD background. This is a traditional method to search for a large resonance signal. It also takes advantage of the fact that the QCD background always produces a smooth and monotonically decreasing spectrum with high statistics that can simply be fit. This methodology makes use of Occam's razor 44 , noting that if the data can be well fit with the background fit function alone, then there is no significant evidence for signal, and the data is compatible with background alone. Hence it is simplest to use the data itself for the background. Nevertheless, most of these searches are aware that this argument has its limitations, for example on the low statistics tail at high dijet mass, and have also compared the data with a QCD Monte Carlo just to confirm that dijet mass data

\footnotetext{
${ }^{\mathrm{c}}$ Integrate both signal and background from $-\cos \theta_{M A X}^{*}$ to $\cos \theta_{M A X}^{*}$ and then solve
} $d(S / \sqrt{B}) / d \cos \theta_{M A X}^{*}=0$. 
are at least roughly compatible with QCD alone. Experimenters have also noted other potential biases when using a parameterization to model the background 45 .

\subsubsection{Data from the CERN S̄ppS Collider Experiments}
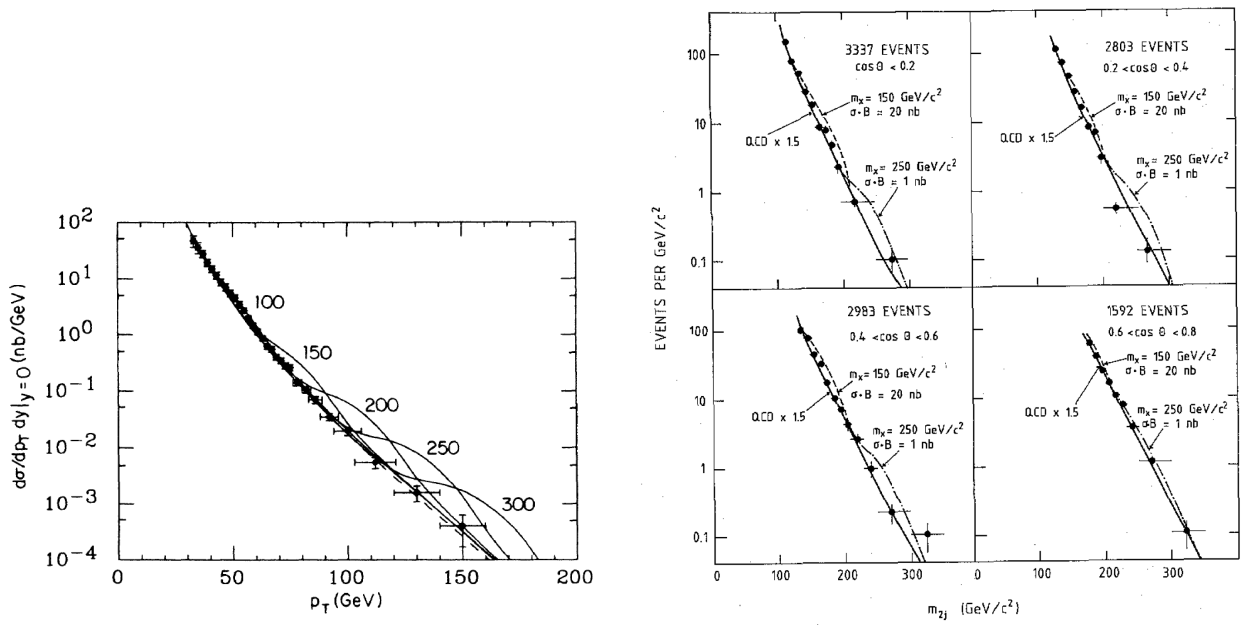

Fig. 6. Data from UA1 in 1986 and 1988. (left) Inclusive jet $p_{T}$ spectrum using $260 \mathrm{nb}^{-1}$, originally from Ref. 24 in 1986, compared to predictions of QCD and axigluons decaying to $N=5$ flavors of quarks from Ref. 12, Copyright 1988 by the American Physical Society. (right) Dijet mass spectra using $490 \mathrm{nb}^{-1}$ in four bins of $\left|\cos \theta^{*}\right|$ compared to predictions of QCD and a vector resonance with width $\Gamma=0.1 M$ from Ref. 25 , Copyright 1988, with permission from Elsevier.

The earliest search for dijet resonances at hadron colliders $24 \mid 12$ was atypical in many respects. In Fig. [6 we reproduce the comparison of UA1 1986 data ${ }^{24}$ from the inclusive jet $p_{T}$ spectrum for $|\eta|<0.7$ with a leading order calculation of QCD plus the axigluon model of a dijet resonance. This comparison was published by theorists 12 in the paper which introduced the theoretical cross section for axigluon production. The analysis was challenging, as the jet $p_{T}$ data had been unsmeared for the effects of jet resolution by the UA1 collaboration, and had to be resmeared by the theorists in order to make a valid comparison with the expected smeared shapes of axigluon resonances in the UA1 detector. Figure 6 also shows a search from UA1 in 1988 12, which used the dijet mass spectrum, and again compared with LO QCD normalized to the data. Both searches using UA1 data required a multiplicative factor of 1.5 to normalize LO QCD to the data. The UA1 1988 data extended to a dijet mass of $0.3 \mathrm{TeV}$ and agreed with the QCD background. The UA1 analysis of the dijet mass spectrum was unique in that it analyzed the data in four bins of $\left|\cos \theta^{*}\right|$ instead of simply applying a single cut on $\left|\cos \theta^{*}\right|$. 

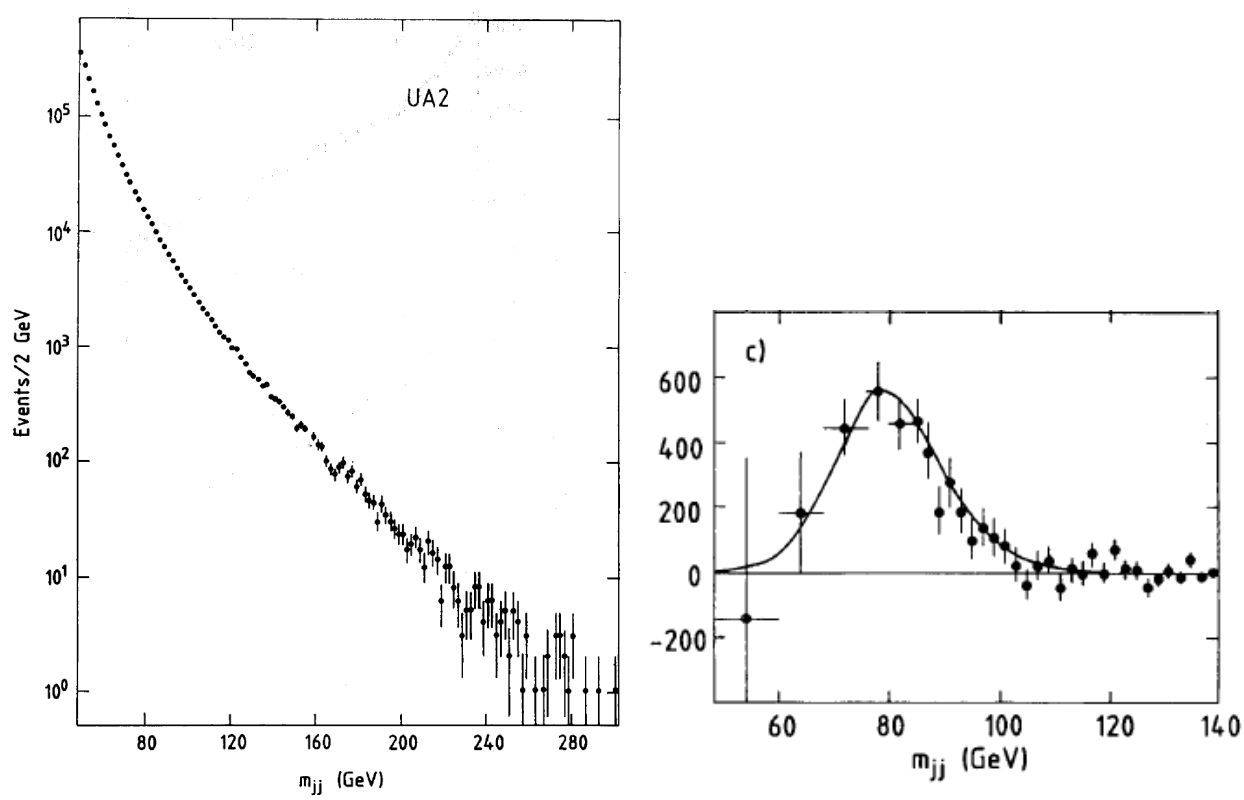

Fig. 7. Data from UA2 in 1990. (left) The dijet mass spectrum using $4.7 \mathrm{pb}^{-1}$ and (right) the difference between the data and the fitted background. The fitted signal from $\mathrm{W}$ and $\mathrm{Z}$ decays to dijets is shown by the solid curve. From Ref. 27] with kind permission from Springer Science+Business Media.

The UA2 collaboration in 1990 published the only observationd in hadron collisions 27 of an $s$ - channel dijet resonance: the $\mathrm{W}$ and $\mathrm{Z}$ boson, whose mass was already known from its previous discovery in decays to leptons. In Fig. 7 we reproduce the dijet mass spectrum before and after the subtraction of the QCD background. The data after the subtraction is well fit by a single peak with shape, accounting for both the $\mathrm{W}$ resonance and the smaller rate for the $\mathrm{Z}$ resonance. The QCD background came from a parameterization of the differential rate as a function of dijet mass, $m$,

$$
\frac{d N}{d m}=\frac{p_{0}}{m^{p_{1}}} e^{-\left(p_{2} m+p_{3} m^{2}\right)},
$$

with four parameters $p_{0}, p_{1}, p_{2}$ and $p_{3}$. The fit of this parameterization to the data in the full dijet mass region $48<m<300 \mathrm{GeV}$ had a probability of only $1 \%$, but excluding the dijet mass region $70<m<100$ that contained the $\mathrm{W}$ and $\mathrm{Z}$ boson, and redoing the fit, gave a probability of $78 \%$. The clear peak after subtracting this second background fit is quite significant, as shown in Fig. 7. The search for the dijet decays of the $\mathrm{W}$ and $\mathrm{Z}$ benefited from apriori knowledge of the existence and

\footnotetext{
${ }^{\mathrm{d}}$ Later observations of $W$ decaying to dijets always involved more complicated processes, such as
} top quark decay. 
the mass of the resonance. Otherwise, the $1 \%$ probability of the first background fit would not be sufficient disagreement with the background to claim a discovery. Nevertheless, this provided an important confirmation of the $\mathrm{W}$ and $\mathrm{Z}$ resonances in the dijet channel. The background parameterization was also used outside the mass region of the $\mathrm{W}$ and $\mathrm{Z}$ to search for higher mass resonances in 1990 27, and again in 199329 . These two papers led the field by introducing searches with parametrized background shapes. It is unfortunate that no figures exist comparing either UA2 analysis for $m>0.12 \mathrm{TeV}$ to the background parameterization]. Both UA2 analyses used dijet mass data in the region $0.05<m<0.3 \mathrm{TeV}$ to search for resonances, with the 1990 search requiring $|\eta|<0.7$ and the 1993 search requiring $\left|\cos \theta^{*}\right|<0.6$.

\subsubsection{Data from the Fermilab Tevatron Collider Experiments}
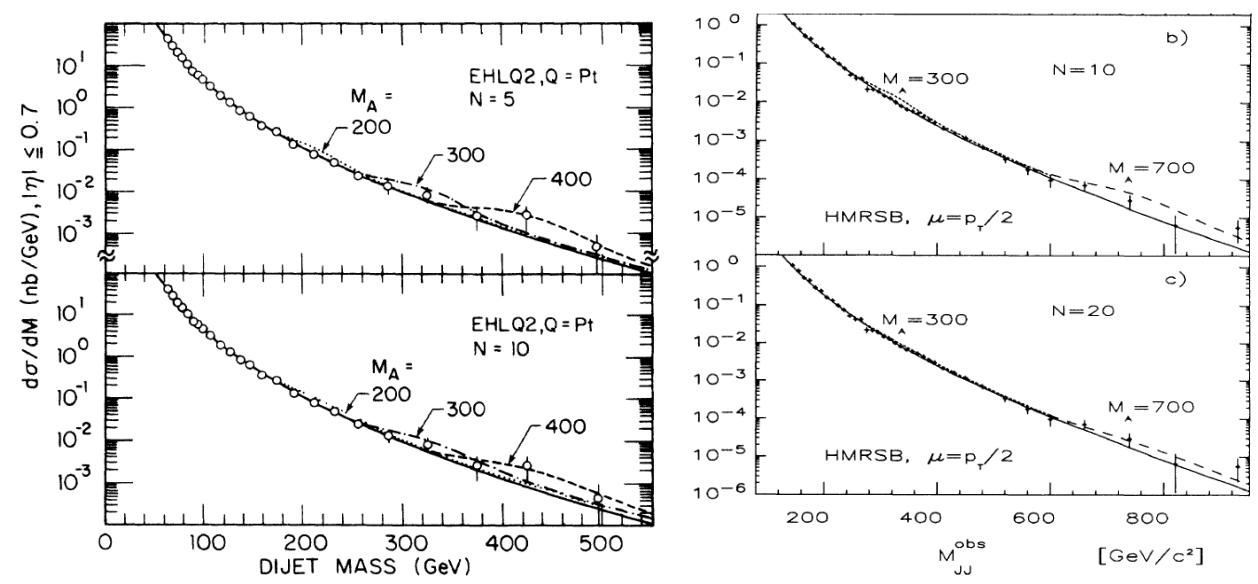

Fig. 8. Data from CDF in 1990 and 1993. (left) Dijet mass spectrum using $26 \mathrm{nb}^{-1}$ compared to predictions of QCD and axigluons decaying to $N=5$ (upper) and $N=10$ (lower) flavors of quarks from Ref. 26] Copyright 1990, and (right) using $4.2 \mathrm{pb}^{-1}$ for $N=10$ (upper) and $N=20$ (lower) from Ref. 28, Copyright 1993 by the American Physical Society.

The two earliest CDF searches in $1990 \underline{26}$ and $1993 \stackrel{28}{28}$ are very similar, and model the background roughly like the UA1 search 25 . Dijet mass data with $|\eta|<0.7$ from these CDF searches are shown in Fig. 8 compared to predictions from the QCD background and axigluon signals. In $1990 \mathrm{CDF}$ searched for dijet resonances, using $26 \mathrm{nb}^{-1}$, with data in the mass interval $60<m<500 \mathrm{GeV}$. In $1993 \mathrm{CDF}$ used 4.2 $\mathrm{pb}^{-1}$ of data to search in the dijet mass interval $140<m<1000 \mathrm{GeV}$. Both CDF analyses modeled the background using LO QCD calculations smeared with the 
dijet mass resolution and normalized to the data at low dijet mass. Both datasets were compatible with the QCD prediction, however, CDF reported in the 1993 paper that " a small excess of events is observed in the data between 350 and 400 $G e V "$ to explain a mass gap in its axigluon exclusions for $N=20$.
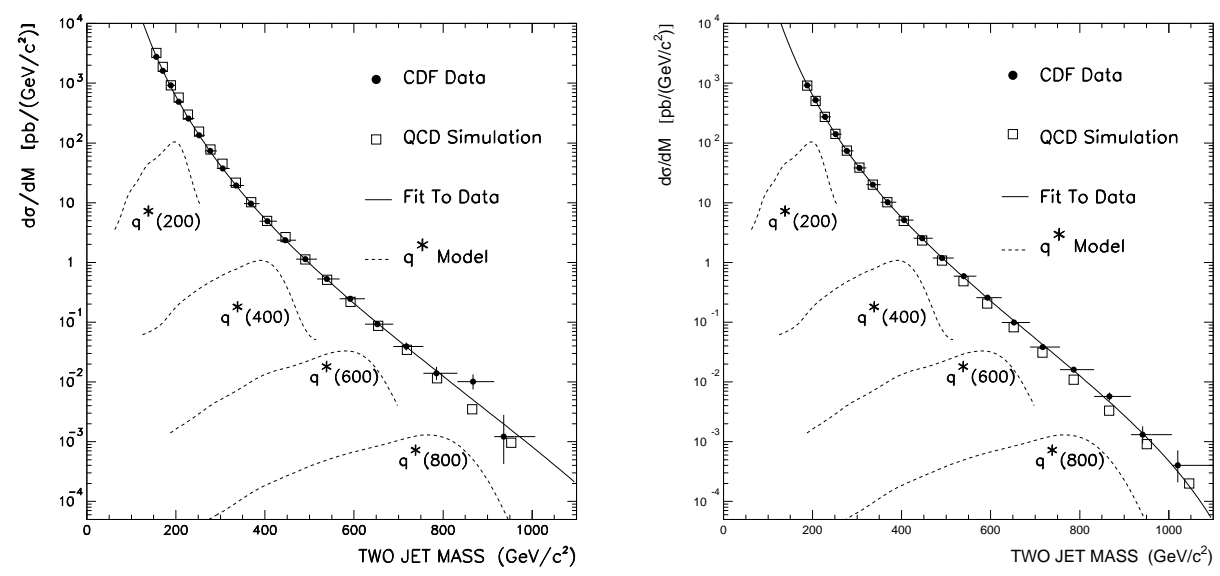

Fig. 9. Data from CDF in 1995 and 1997. (left) Dijet mass spectrum using $19 \mathrm{pb}^{-1}$ compared to a background fit, and simulations of QCD and excited quark signals from Ref. 30] Copyright 1995, and (right) using $106 \mathrm{pb}^{-1}$ from Ref. 31, Copyright 1997 by the American Physical Society.

The CDF searches in $1995 \stackrel{30}{30}$ and 199731 used similar techniques, and modeled the background like the UA2 searches 27|29. Dijet mass data with $|\eta|<2.0$ and $\left|\cos \theta^{*}\right|<2 / 3$ from these CDF searches are shown in Fig. 9. Despite having $5-20$ times more luminosity than the previous search published in 1993, the 1995 and 1997 data also extended to about $m=1 \mathrm{TeV}$ because the $\left|\cos \theta^{*}\right|<2 / 3$ cut suppressed the QCD background. The 1995 search fit the background with a functional form containing only three parameters:

$$
\frac{d \sigma}{d m}=\frac{p_{0}}{m^{p_{1}}}(1-m / \sqrt{s})^{p_{2}}
$$

while the 1997 search used a similar form with four parameters:

$$
\frac{d \sigma}{d m}=\frac{p_{0}}{m^{p_{1}}}\left(1-m / \sqrt{s}+p_{3} m^{2} / s\right)^{p_{2}},
$$

where $\sqrt{s}$ is the $p \bar{p}$ collision energy. These parameterizations were motivated by leading order QCD. The term $m^{p_{1}}$ mimics the mass dependence of the QCD matrix elements (Eq. 17), and was borrowed from the UA2 parameterization in Eq. 46. The term $(1-m / \sqrt{s})^{p_{2}}$ was introduced by CDF and mimics the mass dependence of the parton distributions at an average fractional momentum $x=m / \sqrt{s}$. With increased statistics in 1997 the additional $p_{3}$ term was needed in order to model 
the data at high dijet mass. In addition to the background fit used to search for new physics, CDF also compared their data to a QCD prediction from PYTHIA, with the prediction normalized to the data, on average in 1995, and normalized to the low mass data in 1997. In the 1997 paper CDF noted that the data was "above the QCD simulation at high mass", similar to the excess they reported in the inclusive jet cross section at high $p_{T} 46$. The source of the difference was not understood at the time, but it has since been attributed to the proton PDFs within the QCD prediction. By using a parameterization for the background, CDF was able to minimize the effects of considerable experimental and theoretical uncertainties on the determination of the background shape. In Fig. 10 CDF showed the difference between their data and the background fit, and concluded there was no evidence for new physics. In 1995 they quoted the local significance of fluctuations interpreted as a resonance mass of $250 \mathrm{GeV}(2.3 \sigma), 550 \mathrm{GeV}(1.3 \sigma)$ and $850 \mathrm{GeV}(1.8 \sigma)$, and in 1997 pointed out that the single bin near dijet mass $550 \mathrm{GeV}$ was $2.6 \sigma$ above the fit. It was not common practice in those years to estimate the "look elsewhere effect" 47, used to quote a global significance of observing local fluctuations by also taking into account the probability of observing the fluctuation anywhere in the mass range considered. In Fig. 10 CDF also compared the data with the shape and normalization of excited quark signals, allowing the excluded mass to roughly be estimated by eye directly from the data.
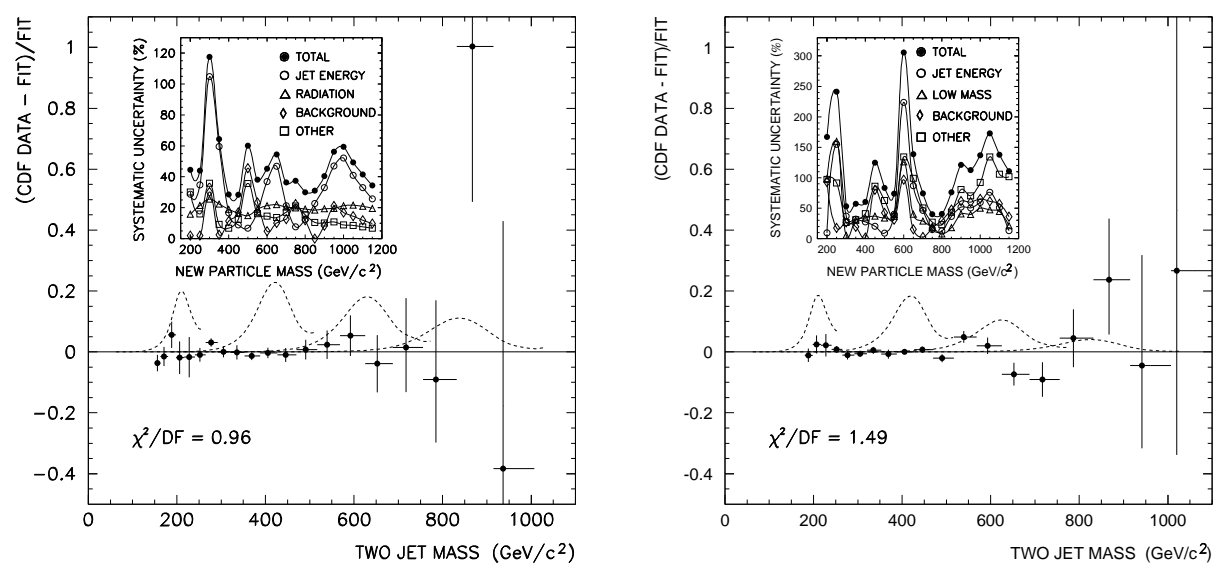

Fig. 10. Fit and systematics from CDF in 1995 and 1997. (left) Fractional difference between data using $19 \mathrm{pb}^{-1}$ and a background fit, compared to simulations of excited quark signals. Inset shows systematic uncertainty for signal. From Ref. 30, Copyright 1995, and (right) using 106 pb$^{-1}$ from Ref. 31 Copyright 1997 by the American Physical Society.

Figure 11 shows D0 data from a search published in 2004 32. The analysis required $|\eta|<1$ and $\left|\cos \theta^{*}\right|<0.67$ and measured dijet masses in the range $0.18<m<1.2 \mathrm{TeV}$. The D0 analysis was unique in using NLO QCD to model the 

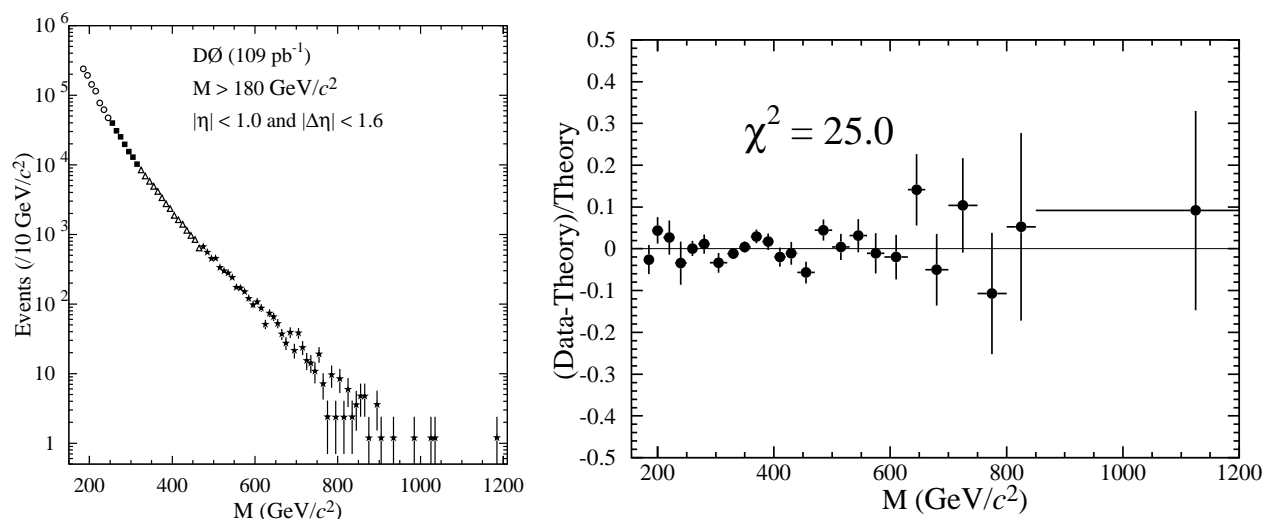

Fig. 11. Data from D0 in 2004. (left) The dijet mass spectrum using $109 \mathrm{pb}^{-1}$ and (right) the fractional difference between the same data and NLO QCD from Ref. [32, Copyright 2004 by the American Physical Society.

background. A QCD calculation from JETRAD 48 with CTEQ6M 49 PDFs and renormalization scale $\mu=0.5 E_{T}^{M A X}$ was smeared with the measured dijet mass resolution. It agreed remarkably well with the data. The $\chi^{2} / D F$ was $25 / 25$, considering only statistical uncertainties and without any change to the normalization of the QCD prediction. Considering the experimental and theoretical uncertainties, the agreement was impressive. This level of agreement between experiment and QCD calculation for the dijet mass distribution, both in shape and in normalization, has not been seen by any of the other experiments.

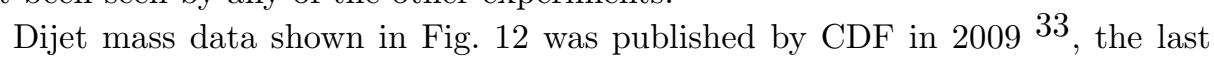
search from the Tevatron. To use the same dataset for both a QCD measurement and a resonance search only $|\eta|<1$ was required, with no cut on $\left|\cos \theta^{*}\right|$, and the mass distribution was measured in the range $0.18<m<1.3 \mathrm{TeV}$. The technique for modeling the background is the same as the 1995 and 1997 CDF searches, however, the following parameterization was developed for an improved fit to the high statistics data over a wide mass range:

$$
\frac{d \sigma}{d m}=\frac{p_{0}(1-m / \sqrt{s})^{p_{1}}}{(m / \sqrt{s})^{\left(p_{2}+p_{3} \ln [m / \sqrt{s}]\right)}} .
$$

This parameterization gave a good fit to the data with $\chi^{2} / D F=16 / 17$, and CDF used it as the background in the search for resonances. In the same paper CDF also compared the data with a full NLO QCD calculation from fastNLO 50 , using CTEQ6.1M 51, and with renormalization scale $\mu=p_{T}^{A V G}$, and found $\chi^{2} / D F=$ $21 / 21$ after taking into account the systematic uncertainties. However, unlike the D0 search, the shape agreement between data and NLO QCD at CDF without including systematic uncertainties was not sufficient to use NLO QCD for the background in a search for resonances. 


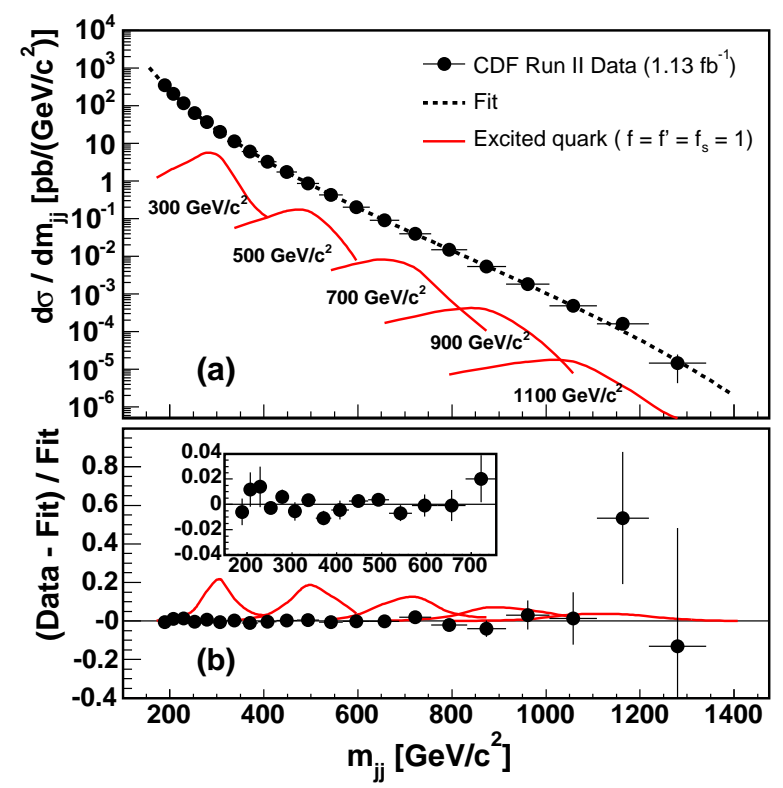

Fig. 12. Data from CDF in 2009. (top) Dijet mass spectrum using $1.13 \mathrm{fb}^{-1}$ compared to a background fit, and simulations of excited quark signals, and (bottom) the fractional difference between the same data and fit and (inset) a zoom at low mass. From Ref. 33 Copyright 2009 by the American Physical Society.

\subsubsection{Data from the CERN Large Hadron Collider Experiments}

The introduction of the Large Hadron Collider greatly accelerated the pace of dijet resonance searches. Data from three ATLAS searches are shown in Fig. 13 , all published within roughly a year. The three searches required $|\eta|<2.5$ and $\left|\cos \theta^{*}\right|<0.57$ for the search in 201034 and winter 201136 , and $|\eta|<2.8$ and $\left|\cos \theta^{*}\right|<0.54$ in summer 201138 . The measured dijet mass range for the three searches was $0.2<m<1.7 \mathrm{TeV}, 0.5<m<2.8 \mathrm{TeV}$, and $0.72<m<4.1$ $\mathrm{TeV}$ respectively. The lowest dijet mass used increased with each search because the jet trigger threshold used for the search increased. All ATLAS searches used the last CDF parameterization in Eq. 49 to model the background. As shown in Fig. 13, the ATLAS searches all reported the bin-by-bin statistical significance of the difference between the data and the background fit, clearly showing that all fits were good and there was no evidence for new physics. The ATLAS searches

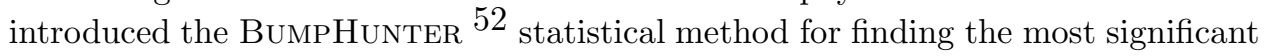
upward fluctuation, and for quantifying its complete global probability, including the "look elsewhere effect", of coming from the background. The largest upward fluctuations and the corresponding background probabilities were $0.55 \mathrm{TeV}$ and at least $51 \%$ in $2010,1.1 \mathrm{TeV}$ and $39 \%$ in winter 2011 , and $1.25 \mathrm{TeV}$ and $82 \%$ in 

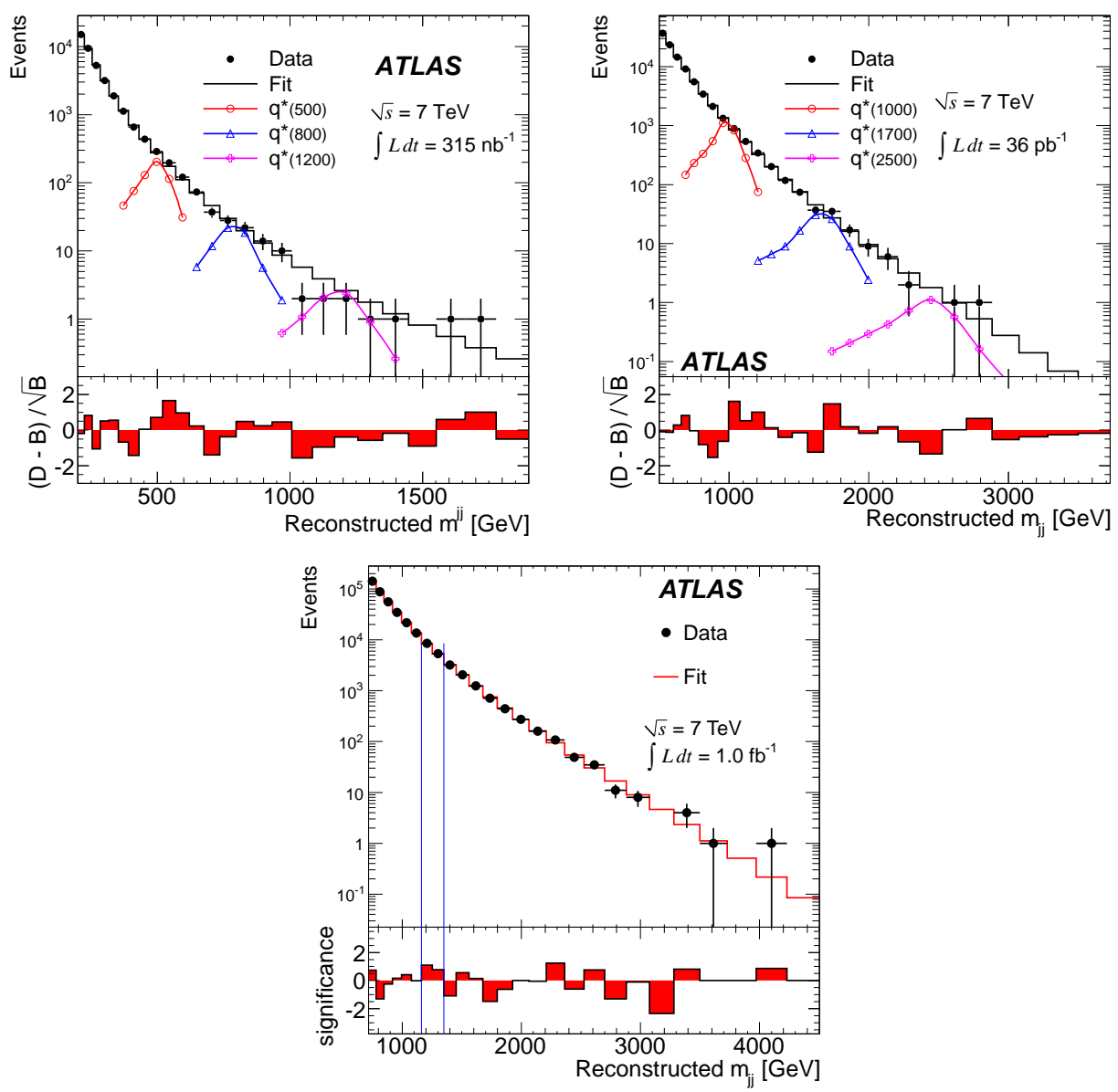

Fig. 13. Data from ATLAS in 2010 and 2011. (top left) Dijet mass spectrum using $315 \mathrm{nb}^{-1}$ compared to a background fit, and simulations of excited quark signals, and the bin-by-bin significance of the difference between the data and the fit from Ref. 34. Copyright 2010 by the American Physical Society. (top right) Same using $36 \mathrm{pb}^{-1}$ from from Ref. 36 (bottom) Same using 1.0 $\mathrm{fb}^{-1}$, and without simulations, from Ref. 38

summer 2011.

The CMS collaboration has published searches for dijet resonances in 201035 and in 2011 37. CMS required $|\eta|<2.5$ and $\left|\cos \theta^{*}\right|<0.57$, the same as the first two ATLAS searches, and measured the dijet mass spectrum in the interval $0.22<m<2.1 \mathrm{TeV}$ in 2010 , and in $0.84<m<3.7 \mathrm{TeV}$ in 2011. In Fig. 14 CMS compared data to both a QCD simulation and to a background fit. The uncertainty in the jet energy scale decreased from $10 \%$ in the 2010 publication to $2.2 \%$ in 2011 , decreasing the uncertainty in the comparison with QCD as shown in Fig. 14. As previously discussed, in 2011 CMS introduced wide jets to improve the resolution for 

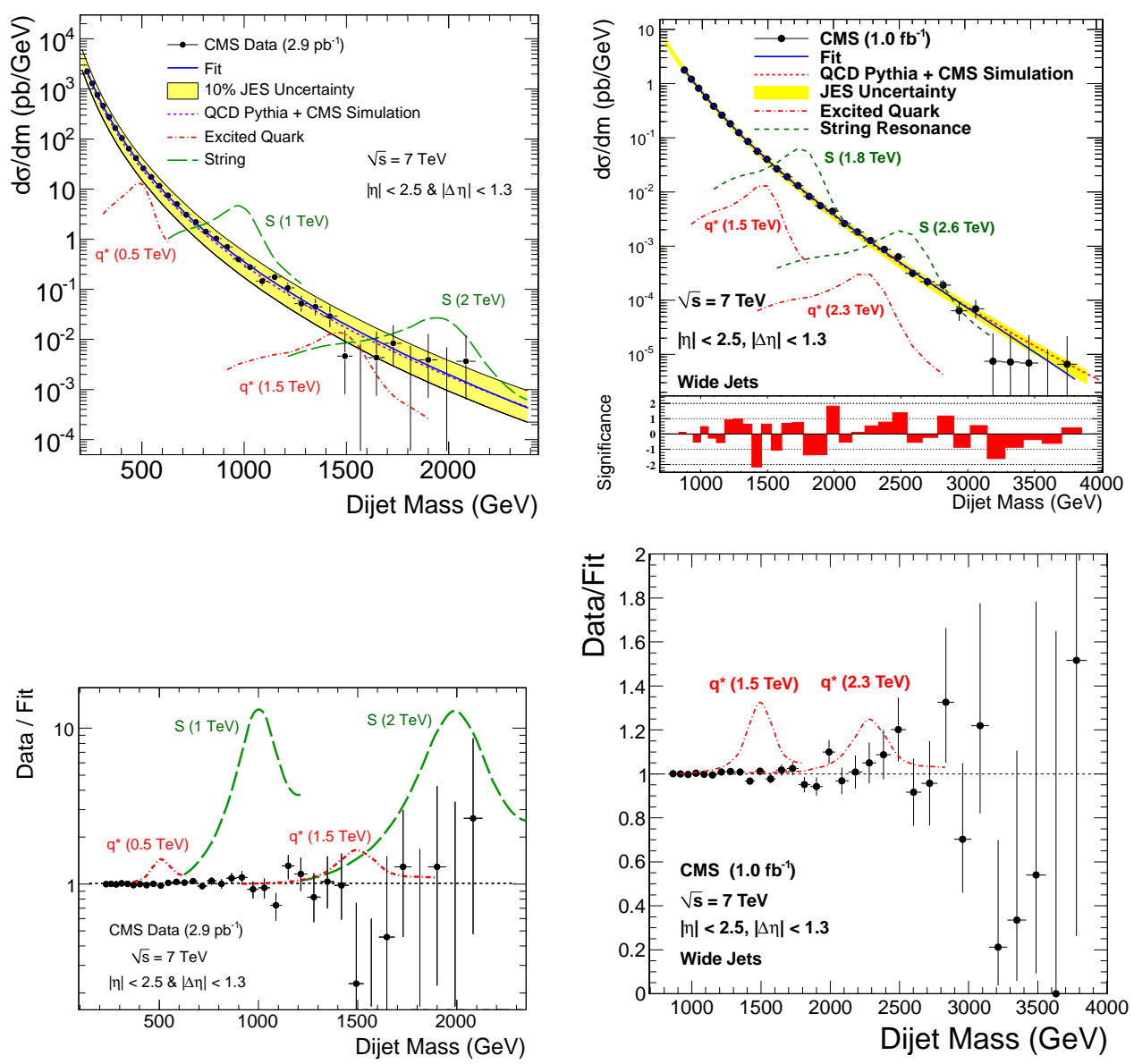

Fig. 14. Data from CMS in 2010 and 2011. (top left) Dijet mass spectrum using $2.9 \mathrm{pb}^{-1}$ compared to a background fit, and simulations of QCD and signals from excited quarks and string resonances. The Band shows the jet energy scale uncertainty. (bottom left) Ratio of the same data to the fit, and compared to the same signals. Left figures from Ref. 35 Copyright 2010 by the American Physical Society. (top right) Same as top left using $1.0 \mathrm{fb}^{-1}$ and including the bin-bybin significance of the difference between the data and the fit. (bottom right) Ratio of the data and the fit compared to excited quark signals. Right figures from Ref. 37 Copyright 2011, with permission from Elsevier.

resonances decaying to gluons. Following the CDF and ATLAS searches, CMS used Eq. 49 to fit the background in the search. The background parameterization gave a good fit: $\chi^{2} / D F=32 / 31$ in 2010 and $\chi^{2} / D F=27.5 / 28$ in 2011. In 2011 CMS adopted the ATLAS style of displaying the bin-by-bin significance of the difference between data and fit, just below the data points, from which one can visually see that there were no significant fluctuations. Unlike CDF and ATLAS, CMS did not quote estimates of the significance of fluctuations in either paper. Following the 
CDF searches, CMS compared their data with the size and shape of various dijet resonance signals in a plot of the data divided by the fit, allowing a direct visual estimate of the signal mass values excluded.

\subsection{Limits and Models}

Limits on dijet resonances are often performed in three steps. The first step is setting limits on the cross section, which can be purely experimental numbers determined using a statistical procedure, and can be fairly independent of any model. The second step is to evaluate the theoretical cross section for the model as a function of resonance mass, which is a completely model dependent process. The third and final step is to compare the experimental upper limit on the cross section, with the theoretical cross section of the model, and find what resonance masses, if any, are excluded. In Table 4 we summarize the mass intervals excluded for the majority of models considered in dijet resonance searches.

Table 4. Excluded mass intervals for resonance models from searches in the dijet mass spectrum. The model parameters used for these exclusions are presented in Section 2.2.10 See the text about each exclusion for any exceptions in the model parameters, discussion of the calculations, and for the additional exclusions of color octet technirhos by CDF and color octet scalars by ATLAS. Continuation rows beginning with a " mark are present when the search excluded multiple mass intervals for a given model.

\begin{tabular}{lccccccc}
\hline Expt. & Year & $\begin{array}{c}\text { Axigluon } \\
\text { or Coloron } \\
(\mathrm{TeV})\end{array}$ & $\begin{array}{c}\text { Excited } \\
\text { Quark } \\
(\mathrm{TeV})\end{array}$ & $\begin{array}{c}W^{\prime} \\
(\mathrm{TeV})\end{array}$ & $\begin{array}{c}Z^{\prime} \\
(\mathrm{TeV})\end{array}$ & $\begin{array}{c}E_{6} \\
\text { Diquark } \\
(\mathrm{TeV})\end{array}$ & $\begin{array}{c}\text { String } \\
(\mathrm{TeV})\end{array}$ \\
\hline UA1 & 1986 & $0.13-0.28$ & - & - & - & - & - \\
UA1 & 1988 & $0.15-0.31$ & - & - & - & - & - \\
CDF & 1990 & $0.12-0.21$ & - & - & - & - & - \\
UA2 & 1990 & - & - & $0.10-0.16$ & - & - & - \\
CDF & 1993 & $0.22-0.64$ & - & - & - & - & - \\
UA2 & 1993 & - & $0.14-0.29$ & $0.13-0.26$ & $0.13-0.25$ & - & - \\
CDF & 1995 & $0.20-0.87$ & $0.20-0.56$ & - & - & - & - \\
CDF & 1997 & $0.20-0.98$ & $0.20-0.52$ & $0.30-0.42$ & - & $0.29-0.42$ & - \\
$"$ & $"$ & & $0.58-0.76$ & & - & - & - \\
D0 & 2004 & - & $0.20-0.78$ & $0.30-0.80$ & $0.40-0.64$ & - & - \\
CDF & 2009 & $0.26-1.25$ & $0.26-0.87$ & $0.28-0.84$ & $0.32-0.74$ & $0.29-0.63$ & $0.26-1.4$ \\
ATLAS & 2010 & - & $0.30-1.26$ & - & - & - & - \\
CMS & 2010 & $0.50-1.17$ & $0.50-1.58$ & - & - & $0.50-0.58$ & $0.50-2.50$ \\
$"$ & $"$ & $1.47-1.52$ & & & & $0.97-1.08$ & \\
$"$ & $"$ & & & & & $1.45-1.60$ & - \\
ATLAS & $2011 \mathrm{w}$ & $0.60-2.10$ & $0.60-2.15$ & - & - & - & - \\
CMS & 2011 & $1.00-2.47$ & $1.00-2.49$ & $1.00-1.51$ & - & $1.00-3.52$ & $1.00-4.00$ \\
ATLAS & $2011 \mathrm{~s}$ & $0.80-3.32$ & $0.80-2.99$ & - & - & - & - \\
\hline
\end{tabular}




\subsubsection{Experimental Systematic Uncertainties}

There are common systematics faced by all the experiments when setting upper limits on the cross section of dijet resonances. Here we give an overview of these systematic uncertainties and their relative importance for the majority of the experiments, before we discuss the limits from each experiment below.

The largest source of experimental systematic uncertainty on the cross-section limits is usually the jet energy scale. This is because the QCD background falls steeply with increasing dijet mass, and the amount of background underneath a potential dijet resonance, at a fixed dijet resonance mass, depends critically on the jet energy scale. For a typical spectrum that falls with a large power of the mass, $d n / d m \sim 1 / m^{N}$, where $N \sim 10$ at high masses, a typical $5 \%$ uncertainty in the jet energy scale leads to an order of $50 \%$ uncertainty in the amount of QCD background underneath the dijet resonance, which increases the cross section limit by roughly $20 \%$ in smooth data samples. When there are upward fluctuations in the data sample the uncertainty of the limit at a nearby resonance mass can be much larger, allowing for the possibility that the fluctuation is attributed to both a resonance and an uncertainty on the jet energy scale. Nearly all experiments correct the jet energy to remove non-linearities and non-uniformities in detector response. There are potentially more serious uncertainties if these jet energy corrections are not continuous, or contain shape deformities, as they create the possibility of manufacturing a bump, or hiding one. The experiments work hard to insure that this does not happen, but they generally do not include any residual uncertainty in a quantitative fashion.

The next largest source of experimental systematic uncertainty is usually the estimation of the QCD background. As discussed in Section 3.2.2, assigning systematic uncertainties is challenging when a QCD calculation is used to model the background. When a simple background parameterization is fit to the data instead, two common methods of evaluating the systematic on the background are: trying alternate parameterizations, or assigning the statistical uncertainty on the fit parameters as a background shape systematic. Either method will frequently give large systematics on the parametrized background in the region of high dijet mass, where the small amounts of data are not sufficient to constrain the background fit.

Most experiments report that the uncertainty on the jet energy resolution does not have a significant effect on the limits, and in particular is often significantly smaller than the effect of the jet energy scale uncertainty. While this may seem surprising at first, it can be understood in the same way that the effect of the jet energy scale was quantified. On a steeply falling QCD dijet mass spectrum, a percentage shift in the mass will have a much larger effect on the amount of background underneath the resonance, compared to roughly the same percentage widening of the Gaussian resolution. The counter example would be a constant background, flat as a function of dijet mass, where a percentage shift in the jet energy scale would have no effect on the amount of background underneath the 
peak, while the same percentage widening of the jet energy resolution would be more important. As a conclusion, the resolution uncertainties are negligible because the QCD background is falling steeply.

The astute reader should note that the arguments above are relevant for the limits on dijet resonances, which is what most of the searches have reported, and the effect of systematic uncertainties would be different if a signal were observed.

\subsubsection{Limits from the CERN S $\bar{p} p S$ Collider Experiments}

The first published exclusion 12 which used UA1 data 24 did not use a statistical method, or discuss systematic uncertainties. Instead a purely visual exclusion was performed. The left plot of Fig. 6 was presented overlaying the observed jet $p_{T}$ distribution with that from QCD plus the axigluon model. The paper states "We see . . that axigluons in the range $M_{A}=125-275 \mathrm{GeV}$ are ruled out" and "... are in contradiction with the data."

The search from UA1 in 1988 was the first to exclude cross sections for resonances at $95 \% \mathrm{CL}$, and used the method of maximum likelihood in a fit of the QCD background prediction and the resonance shapes to the data. The UA1 jet energy scale uncertainty was reported as $9 \%$, however, UA1 used the agreement between LO QCD and the shape of the UA1 data at low dijet mass to constrain the jet energy scale uncertainty for this search to less than $6 \%$ at $95 \%$ CL, and then included a possible $6 \%$ increase in the jet energy scale into the limit. UA1 also noted that uncertainties in the background estimate from higher order QCD processes were not included and could weaken the reported limits. The upper limits shown in Fig.15 were found for narrow resonances $(\Gamma<0.1 M)$ and wide resonances $(\Gamma<0.4 M)$, with the limits corrected for the acceptance of the decay's angular distribution, for the two cases of scalar and vector resonances. UA1 then compared the wide vector resonance cross-section upper limit to the model cross section from the previous search ${ }^{12}$ in Fig. 15 to exclude axigluons in the mass range $150<m_{A}<310 \mathrm{GeV}$.

In 1990 UA2 27 reported upper limits on the cross section shown in Fig. 15, Here limits at $90 \%$ CL were obtained from a fit to the background parameterization and a signal, after subtracting the fitted signal for the $\mathrm{W}$ and $\mathrm{Z}$ resonances. A systematic uncertainty on the cross-section upper limit, as much as $21.5 \%$, came from the dijet mass resolution uncertainty, which was the dominant systematic. UA2 reduced the jet energy scale uncertainty to give only an $11 \%$ uncertainty on the cross-section upper limit, by calibrating the jets with the observed $\mathrm{W}$ and $\mathrm{Z}$ peak. UA2 compared the upper limits on the cross section to an $O\left(\alpha_{s}^{2}\right)$ calculation of the $W^{\prime}$ resonance with standard model couplings to exclude the resonance decays in the mass interval $101<M<158 \mathrm{GeV}$.

The search from UA2 in $1993^{29}$ fit a dijet resonance signal and the background parameterization in Eq. 46 to the data, obtaining the signal cross section and its error. From the best-fit signal cross section and its error UA2 calculated the $90 \% \mathrm{CL}$ upper limit including statistical uncertainties only. The systematics were included in 

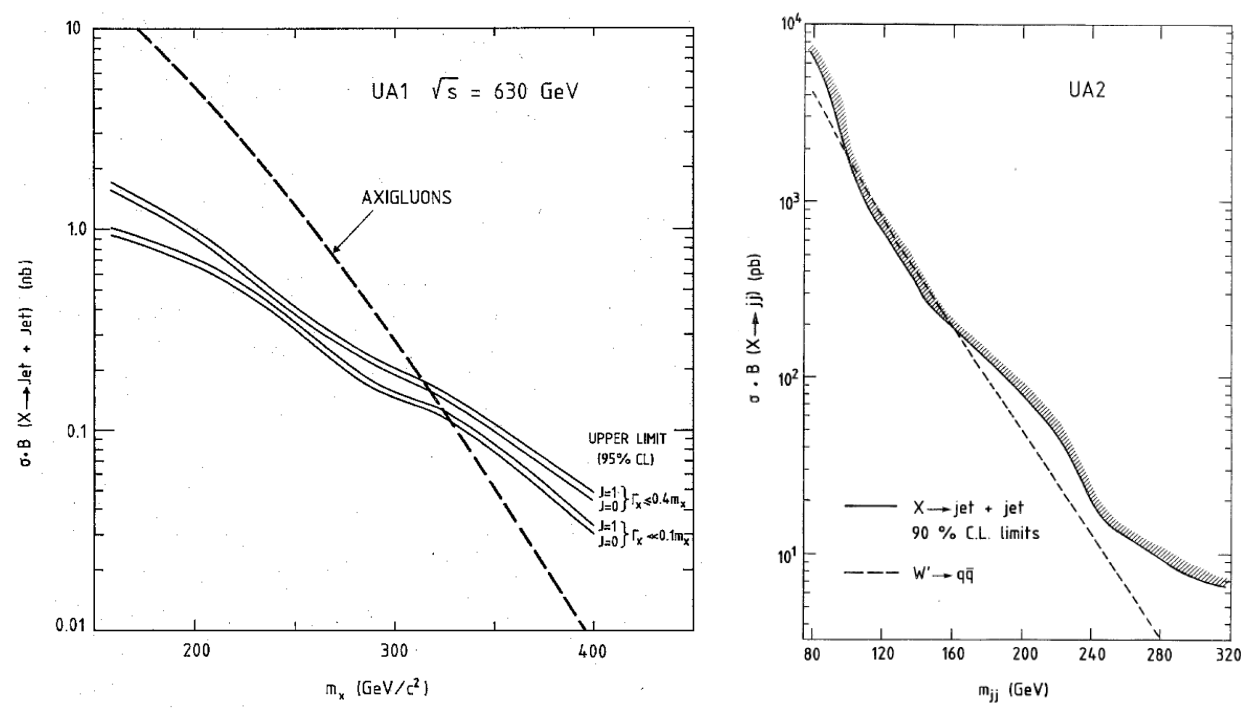

Fig. 15. Limits from UA1 in 1988 and UA2 in 1990: (left) Upper limits at 95\% CL on dijet resonance cross section times branching ratio from UA1 using $490 \mathrm{nb}^{-1}$, for both vector and scalar particles of two different widths, compared to an axigluon calculation. From Ref. 25. Copyright 1988, with permission from Elsevier. (right) Upper limits at 90\% CL on dijet resonance cross section times branching ratio from UA2 using $4.7 \mathrm{pb}^{-1}$, compared to a $W^{\prime}$ calculation. From Ref. 27] with kind permission from Springer Science+Business Media.
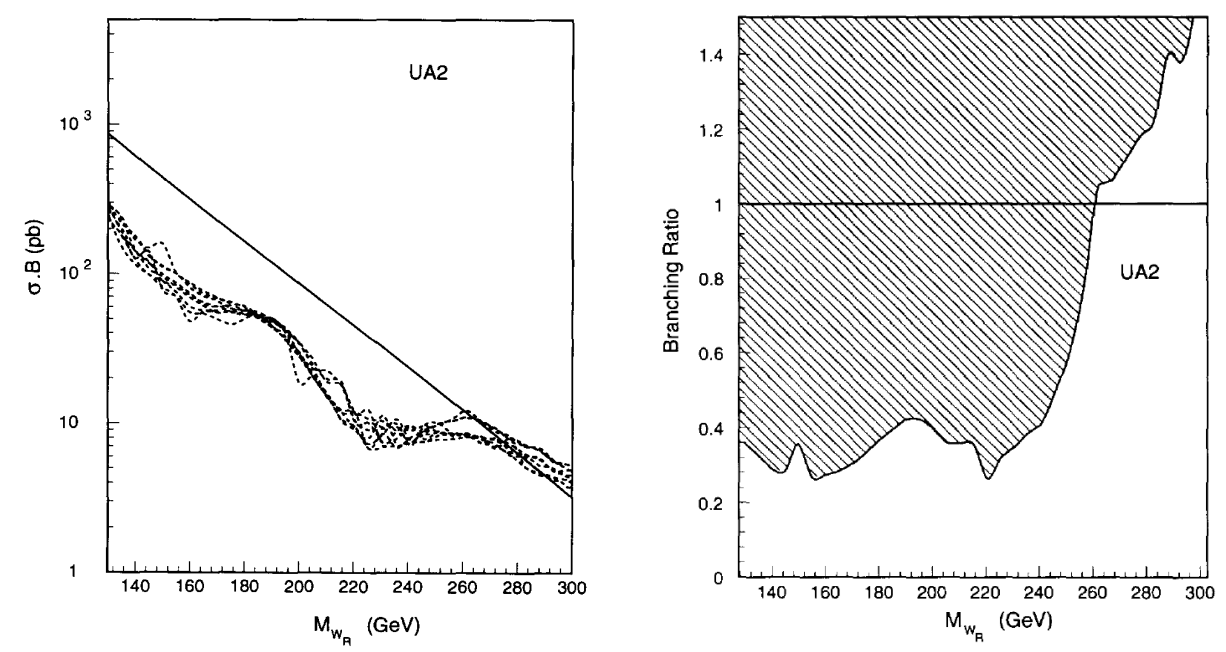

Fig. 16. Limits from UA2 in 1993. (left) Upper limits at 90\% CL on dijet resonance cross section times branching ratio using $11 \mathrm{pb}^{-1}$, with the nine curves showing systematic variations in the limit, compared to a $W^{\prime}$ cross section calculation, and (right) the excluded mass region for a $W^{\prime}$ in the mass vs. branching ratio plane, from Ref. 29] Copyright 1993, with permission from Elsevier. 
a second step and consisted of the uncertainty on the dijet resonances decay width, and the uncertainty on the stability of the dijet mass measurement between the two data samples used from the 1989 and 1990 running periods. For each quantity, $x$, with uncertainty $\Delta x$, they performed the cross-section fit with the three different values $x, x+\Delta x$ and $x-\Delta x$. Therefore, for the two systematics considered, there were nine independent fits and nine different values of the cross-section upper limit. For a $W^{\prime}$ signal, Fig. 16 shows the cross-section upper limit from the nine fits. UA2 selected the largest value among the nine as the upper limit on the cross section including systematics, and used that to exclude at 90\% CL the $W^{\prime}$ in the mass region $130<M<261 \mathrm{GeV}$. Also in Fig. 16 UA2 presented their excluded region in the branching ratio vs. mass plane of the $W^{\prime}$, where a branching ratio of 1 represents decays to $u d$ and $c s$ quarks, as expected from a right handed $W^{\prime}$ model, $W_{R}$. This was the only exclusion of a $W_{R}$ model in dijet resonance searches, as all other searches were for a more standard model like left-handed $W^{\prime}$. Using similar techniques, UA2 also excluded at $90 \%$ CL $Z^{\prime}$ bosons decaying to dijets in the mass region $130<M<252 \mathrm{GeV}$, and mass degenerate excited quarks $\left(q^{*}\right)$ in the mass region $140<M<288 \mathrm{GeV}$. Fig. 18 shows the UA2 $q^{*}$ limits in the coupling vs. mass plane. UA2 also presented mass limits for a few other variations of the $W^{\prime}$, $Z^{\prime}$, and $q^{*}$ models 29. The cross section of the $W^{\prime}$ and $Z^{\prime}$ models came from an $O\left(\alpha_{s}^{2}\right)$ NNLO calculation, and the cross section for the $q^{*}$ model came from a LO Born level calculation.

\subsubsection{Limits from the Fermilab Tevatron Collider Experiments}

The search from CDF in $1990 \frac{26}{26}$ did not publish upper limits on the cross section. A $\chi^{2}$ was calculated for a fit of an axigluon plus LO QCD background to the data, and a jet energy scale uncertainty ranging from $5 \%$ to $9 \%$ was included by allowing both edges of each dijet mass bin to vary, and CDF used the smallest resulting $\chi^{2}$ to exclude axigluons with $N=5$ decay channels in the mass range $120<m_{A}<210$ $\mathrm{GeV}$ at $95 \% \mathrm{CL}$. In $1993 \mathrm{CDF} 28$ published a table of upper limits on the cross section, the first example of publishing generic upper limits, but did not compare them with any models in a figure. The 1993 paper directly excluded axigluons with $N=10$ decay channels in the mass range $220<m_{A}<640 \mathrm{GeV}$ at $95 \% \mathrm{CL}$, with an unspecified statistical test. The paper also set limits on axigluons with $N=20$ decay channels, and presented limits for two choices of PDFs. Both the 1990 and 1993 papers from CDF used a coherent sum of the axigluon signal and the LO QCD background, normalized to the data at low dijet mass.

In $1995^{30}$ and $1997^{31}$ CDF published cross-section upper limits on dijet resonances. The statistical technique was described in the prior CDF search for excited quarks in the $\gamma+$ jet and $W+$ jet channel 53 . Using a binned likelihood for each value of resonance mass, the best fit to the data of the parametrized background plus a floating signal were found. For this background the binned likelihood, $L$, was written as a function of the signal normalization $\alpha$ : 

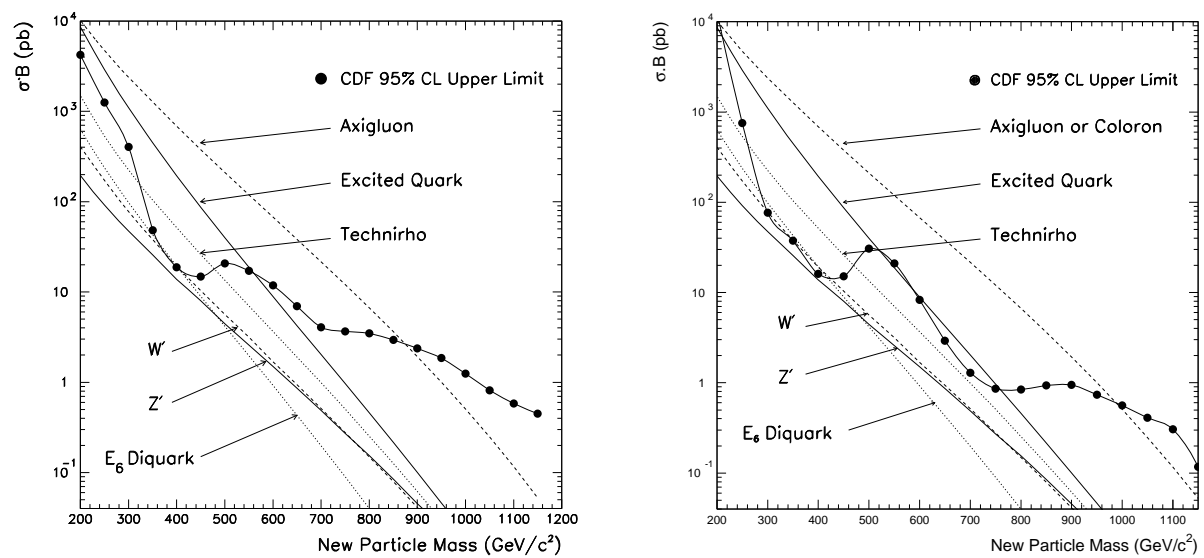

Fig. 17. Limits from CDF in 1995 and 1997. (left) Upper limits at 95\% CL on dijet resonance cross section times branching ratio times acceptance using $19 \mathrm{pb}-1$, compared to calculations of the same observable in six models, from Ref. 30] Copyright 1995, and (right) the same using 106 $p b^{-1}$, from Ref. 31 Copyright 1997 by the American Physical Society.

$$
L=\prod_{i} \frac{\mu_{i}^{n_{i}} e^{-\mu_{i}}}{n_{i} !} \quad \text { where } \quad \mu_{i}=\alpha n_{i}(S)+n_{i}(B) .
$$

Here $n_{i}$ is the number of events observed in the data in dijet mass bin $i, n_{i}(S)$ is the number of events predicted for the signal using the resonance shape in the same bin, and $n_{i}(B)$ is the number of events predicted for the background in the same bin. The background parameterization used in 1995 was Eq. 47 and in 1997 it was Eq. 48. The 95\% quantile of the likelihood distribution in Eq. 50 was found, defining an upper limit on the signal cross section with statistical uncertainties only. The dominant sources of systematic uncertainty considered by CDF were the $5 \%$ jet energy scale uncertainty, the QCD radiation effects on the the mass resonance line shape, and the background parameterization, while other sources were also included. For each $1 \sigma$-shift in the source of systematic uncertainty, the background plus signal fit was repeated, giving a new limit. The change in the limit defined a $1 \sigma$ uncertainty in the signal cross section for each source of systematic, and a total systematic was derived by adding these in quadrature, as shown in the insets in Fig. 10. The likelihood distribution of Eq. 50 was then convolved with the total Gaussian systematic uncertainty in the cross section, and the final limit including systematics was defined as the $95 \%$ quantile of this smeared likelihood. The crosssection upper limits on narrow dijet resonances are shown in Fig. 17 where the effect of the small upward fluctuation in the data near a dijet mass of $550 \mathrm{GeV}$ in both publications produced a noticeable bump in the upper limit. The upper limits were published in a table, and can be compared to the cross section for any model 
of dijet resonances decaying to two partons with $|\eta|<2$ and $\left|\cos \theta^{*}\right|<2 / 3$. CDF explicitly did the comparison with a few model cross sections, shown in Fig. 17 and the reader can note that the strong processes, like $q^{*}$, have much larger cross sections than the weak processes like $W^{\prime}$. From this figure CDF excluded in 1995 and 1997 the mass intervals listed in table 4 for axigluons, excited quarks, $W^{\prime}, Z^{\prime}$ and $E_{6}$ diquarks. CDF also excluded color octet technirhos in the mass interval $320<M<480 \mathrm{GeV}$ in 1995 and $260<M<470 \mathrm{GeV}$ in 1997. CDF used its own LO calculations of the cross section for all models 54 , and included $O\left(\alpha_{s}\right) K$-factors for the $W^{\prime}$ and $Z^{\prime}$ models. The calculations for axigluons, $W^{\prime}, Z^{\prime}$, and $E_{6}$ diquarks employed the narrow width approximation discussed in section 2.2.11 In 1997 CDF noted that the cross section for the coloron model was always greater than or equal to that for the axigluon model, and so all the 95\% CL upper limits for axigluons also applied to colorons. For the excited quark model, CDF plotted the exclusion in the coupling vs. mass plane in Fig. 18, comparing with the dijet resonance search from UA2 in 1993. Figure 18 also shows prior CDF exclusions of $q^{*}$ decays from the $\gamma q$ and $W q$ channel, which were combined with the limit from the dijet channel in 1995 to extend the $q^{*}$ mass limit from $560 \mathrm{GeV}$ with dijets alone to $570 \mathrm{GeV}$ including $\gamma q, W q$ and $g q$ decays.
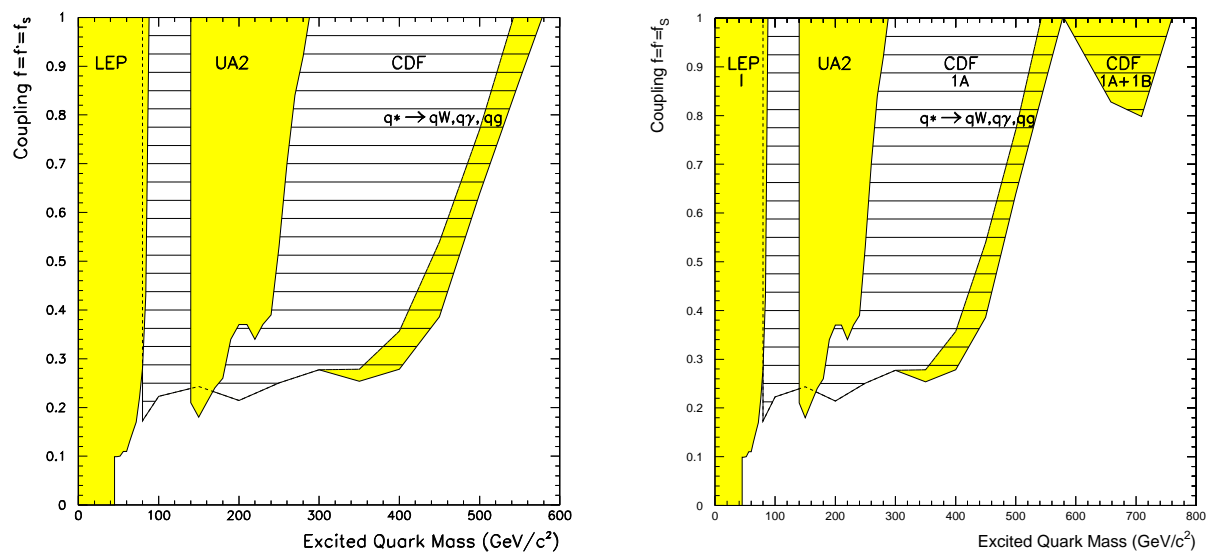

Fig. 18. Excited quark excluded regions from CDF in 1995 and 1997 including UA2 from 1993. (left) Region of the excited quark coupling vs. mass plan excluded by CDF using $19 \mathrm{pb}-1$ in the $q g$ channel, combined with exclusions in the $q W$ and $q \gamma$ channel, and compared with the dijet exclusions from UA2 in 1993 and also from LEP, from Ref. 30. Copyright 1995, and (right) same using $106 \mathrm{pb}-1$, from Ref. 31. Copyright 1997 by the American Physical Society.

The search from D0 in $2004 \frac{32}{32}$ published limits on three models of dijet resonances: $W^{\prime}, Z^{\prime}$ and $q^{*}$. The handling of statistical uncertainties in the limit was similar to the technique used by CDF in 1995 and 1997, which D0 noted as a Bayesian technique with a flat prior for the signal 55 . D0, unlike CDF, applied a 
truly Bayesian methodology to the treatment of the systematic uncertainties. The systematics considered were the jet energy scale $(2 \%)$, resolution, efficiency, and luminosity, with no uncertainty on the background from the NLO QCD calculation. All these nuisance parameters had Gaussian prior probability distributions with widths given by their uncertainties. Eq. 50 was multiplied by all priors, and then integrated over the nuisance parameters to obtain the posterior probability density as a function of the amount of signal, from which the limit was found as the $95 \%$ quantile. This relatively modern procedure likely resulted in a smaller and more correct effect of the experimental systematic uncertainties on the limit than the conservative procedure used by CDF in 1995 and 1997. In Fig. 19 the upper limits at $95 \%$ CL on cross section time branching fraction times acceptance are shown separately for each of the three models, and compared to the model cross sections. D0 obtained the $q^{*}, W^{\prime}$ and $Z^{\prime}$ LO cross sections from PyтнIA, and also applied NLO correction factors of about 1.3 to the $W^{\prime}$ and $Z^{\prime}$. The mass intervals excluded for the the three models are listed in table 4. Comparing to the CDF limits from 1997, which used a similar sized dataset from the same running period, we note that D0 was able to obtain a $Z^{\prime}$ mass limit while CDF was not, D0 $W^{\prime}$ mass limits were significantly better than the corresponding CDF mass limits, and the D0 $q^{*}$ mass limit was a little better than CDF and filled in the CDF gap in the $q^{*}$ mass limit around $550 \mathrm{GeV}$. Comparisons of mass limits are perilous, as the model cross sections presented by the two experiments do not agree. For example, at a resonance mass of $700 \mathrm{GeV}$, where the acceptance of the two experiments was the same, the D0 $W^{\prime}$ model cross section in Fig. 19 is around 1.3pb, about a factor of 2.6 times the CDF $W^{\prime}$ model cross section, and equal to the D0 $q^{*}$ model cross section at the same mass. The upper limits on the cross section are generally better to compare than mass limits, particularly in regions where the acceptance is the same, and the D0 cross-section upper limits at $700 \mathrm{GeV}$ in Fig. 19 are around $0.8-1 \mathrm{pb}$ for all three models which is better than the CDF limit of $1.3 \mathrm{pb}$.

The search from CDF in $2009 \stackrel{33}{33}$ published upper limits on the cross section for dijet resonances. Similar to the 1997 search, CDF also applied a Bayesian methodology starting from Eq. 50 for the binned likelihood including statistical uncertainties only. Unlike the CDF 1997 search but similar to the D0 search, the method for incorporating systematic uncertainties was truly Bayesian 56. The systematic uncertainties considered were the jet energy scale (2-3\%), resolution, and luminosity. CDF used the parameterization in Eq. 49 to describe the background. To account for systematic uncertainties in the background, CDF utilized a profile likelihood method, in which for each value of the signal cross section considered the parameters of the background were found again by maximizing the likelihood. This is in contrast to the previous CDF analysis in 1997, where the background parameters were held fixed as the signal cross section was varied. CDF used the shapes from four models of dijet resonances $W^{\prime}, Z^{\prime}$, RS graviton, and $q^{*}$, available in PYTHIA, to set upper limits at $95 \%$ CL on the cross section and noted that the limits get progressively worse as more gluons are included in the final state. CDF then compared 

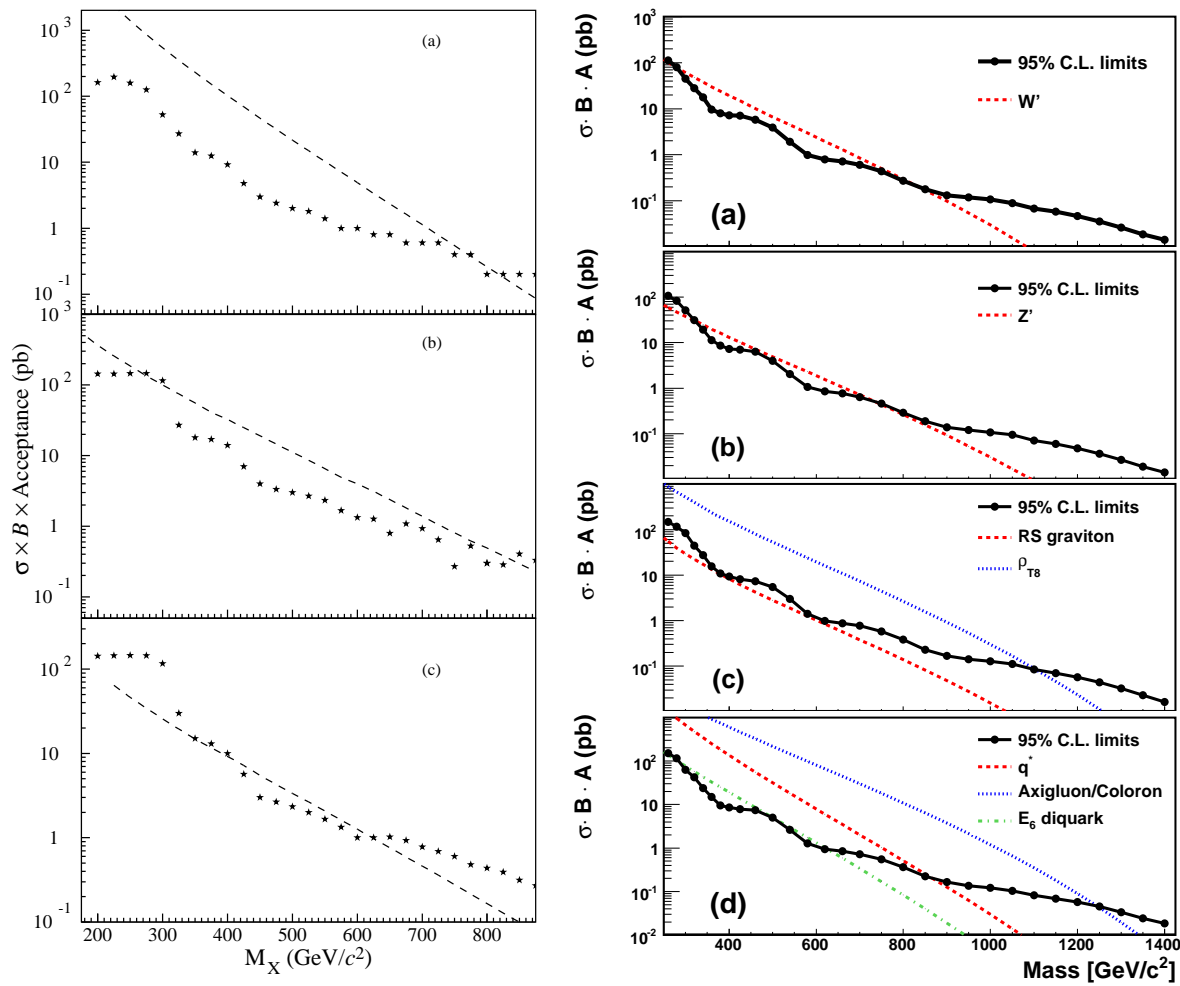

Fig. 19. Limits from D0 in 2004 and CDF in 2009. (left) Upper limits at 95\% CL on dijet resonance cross section times branching ratio times acceptance from D0 using $109 \mathrm{pb}^{-1}$, compared with three model calculations and using resonance shapes from three models: a) excited quarks, b) $W^{\prime}$, c) $Z^{\prime}$, from Ref. 32. Copyright 2004, and (right) same from CDF using $1.13 \mathrm{fb}^{-1}$, compared with seven model calculations and using resonance shape from four models: a) $W^{\prime}$, b) $Z^{\prime}$, c) RS graviton, and d) excited quarks, from Ref. 33 Copyright 2009 by the American Physical Society.

these upper limits to the model cross sections for these four models, from PYTHIA, and to its own LO calculations 54 of the cross section for color octet technirhos, axigluons, colorons, and $E_{6}$ diquarks in Fig. 19, The resulting mass limits are shown in Table 4 for most models. The CDF cross-section calculations for axigluons, colorons, and $E_{6}$ diquarks used the same technique as in its 1997 search, and CDF included the customary NLO k-factor of 1.3 for the $W^{\prime}$ and $Z^{\prime}$ models. The color octet technirho cross section came from PyтніA, where the model included the QCD background, and which had to be subtracted off in order to obtain the net resonance cross section 54 . CDF excluded color octet technirhos in the mass interval $260<M<1100 \mathrm{GeV}$ in 2009. No mass limits were set on Randall-Sundrum gravitons by this search, or by any other dijet resonance search up to this date. CDF presented the upper limits in a table for future use, which allowed the CMS experiment to compare the CDF upper limits on the cross section to that expected 
for a string resonance and determine a CDF mass limit of $1.4 \mathrm{TeV}$ on string resonances 35. In $2009 \mathrm{CDF}$ published the most stringent mass limits to date on color octet technirhos and the dijet decays of $Z^{\prime}$.

\subsubsection{Limits from the CERN Large Hadron Collider Experiments}
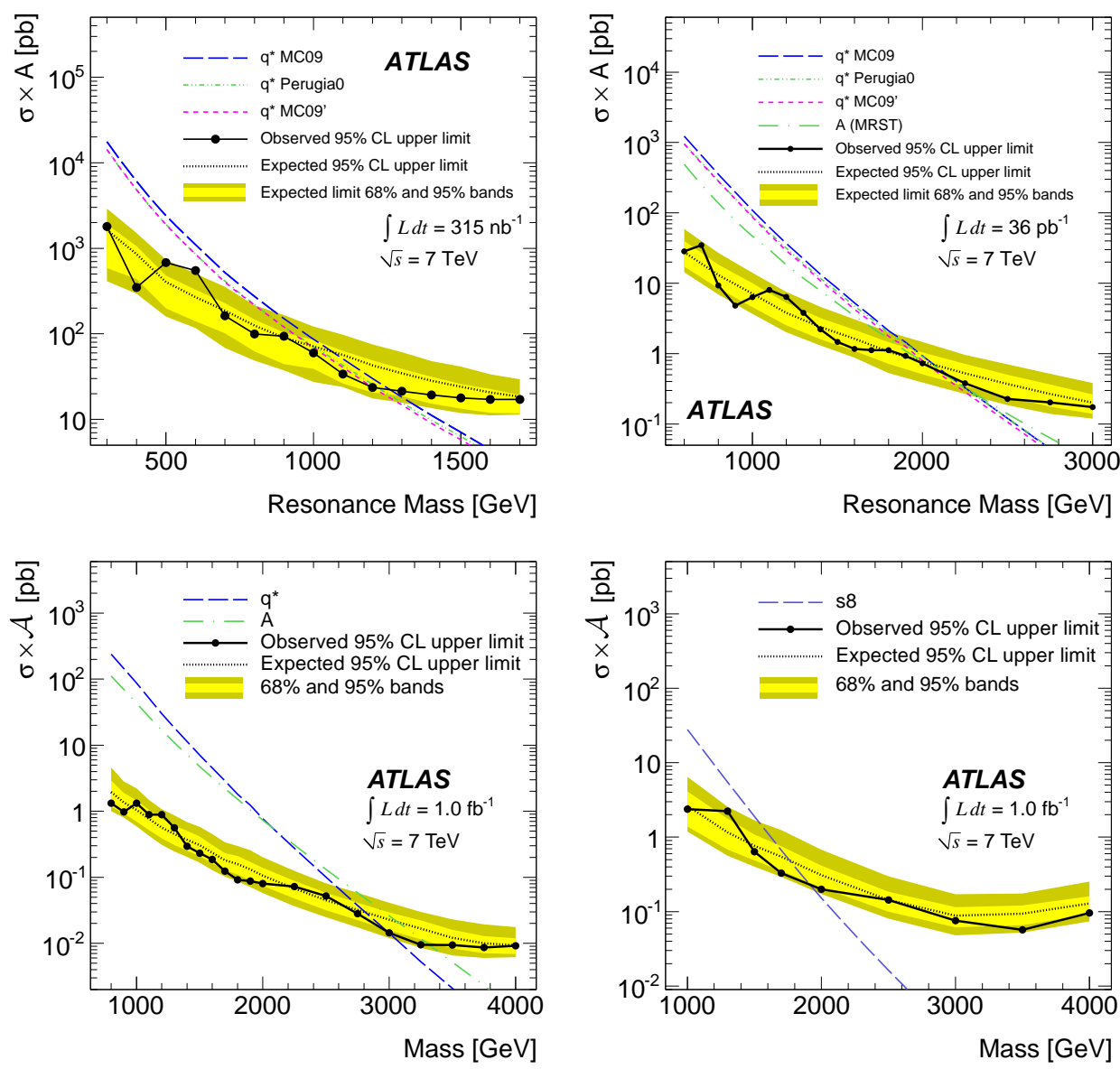

Fig. 20. Limits from ATLAS in 2010, winter 2011, and summer 2011. (top left) Observed and expected upper limits at $95 \%$ CL on excited quark cross section times acceptance using $315 \mathrm{nb}-1$, compared to predictions of excited quarks with various tunes and PDFs. Shaded bands are $1 \sigma$ and $2 \sigma$ variations in the expected limit. From Ref. 34. Copyright 2010 by the American Physical Society. (top right) Same using $36 \mathrm{pb}^{-1}$ and in addition comparing to an axigluon prediction. From Ref. 36 (bottom left) Same using $1.0 \mathrm{fb}^{-1}$ and (bottom right) same, except limits and predictions are specifically for a color octet scalar resonance decaying to $g g$, from Ref. 38.

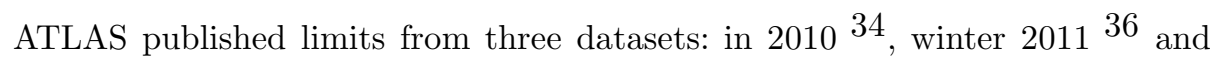


summer 201138 , with $0.3,36$, and $1000 \mathrm{pb}^{-1}$ of integrated luminosity. Limits were set using a Bayesian statistical technique with uniform prior for the signal cross section, and systematic uncertainties were incorporated in a fully Bayesian treatment using Gaussian priors. The dominant source of systematic uncertainty was the jet energy scale in all three publications: $6 \%-9 \%$ in $2010,3.2 \%-5.7 \%$ in winter 2011, and less than $4 \%$ in summer 2011. The background was determined using the last CDF parameterization in Eq. 49. The systematic uncertainty on the background was taken from the statistical uncertainty in the fitted parameters, and for the winter 2011 search the uncertainty increased with resonance mass from 3\%$40 \%$. The luminosity uncertainty was $11 \%, 11 \%$, and $3.7 \%$ for the three searches respectively. In all three searches the uncertainty in the jet energy resolution had a negligible effect on the limits.

ATLAS published the first dijet resonance searches that included expected limits and their variation at the $1 \sigma$ and $2 \sigma$ levels. These are limits determined from pseudoexperiments generated from the smooth background prediction. Expected limits vary smoothly as a function of resonance mass, while observed limits have wiggles that reflect statistical fluctuations in the data. In Fig. 20 the ATLAS observed and expected limits at $95 \% \mathrm{CL}$ are compared with the calculations of the model cross sections. In 2010 the fluctuation at $0.55 \mathrm{TeV}$ mentioned previously produced a $2 \sigma$ fluctuation in the observed limit, their largest upward fluctuation.

In all three publications ATLAS compared their cross-section upper limits from a $q^{*}$ shape to the cross section times acceptance for the $q^{*}$ model from PYTHIA including all decays: dominantly $q g$, but also including roughly an additional $20 \%$ "dijet" cross section resulting from $q W, q Z$, and $q \gamma$. ATLAS explored variations in the $q^{*}$ cross section with different Monte Carlo tunes and associated PDFs, and chose to quote as the main result the MC09 tune 57 with MRST2007 PDF 58 . In 2011 ATLAS also compared its cross-section upper limits to an axigluon calculation using CALCHEP 59, again with MRST2007 PDF. We discuss the ATLAS calculations more in Appendix A. The excluded mass intervals for $q^{*}$ and axigluons are listed in table 4 and the expected and observed mass limits are compared in table 5. In the summer of 2011 ATLAS set cross-section upper limits on color octet scalars, which decay to $g g$ and have a wider line shape than $q^{*}$ or axigluons. ATLAS compared with the model cross section from a MADGRAPH 60 plus PYTHIA calculation to exclude color octet scalars in the mass interval $1.0<M<1.92 \mathrm{TeV}$ when the mass limit $1.77 \mathrm{TeV}$ was expected. All observed mass limits from ATLAS are larger than their expected mass limits, due to downward fluctuations in the data. ATLAS in the summer of 2011 has published the most stringent limits to date on axigluons, excited quarks and color octet scalars.

In 2011 ATLAS introduced generic upper limits for wide dijet resonances based on a Gaussian line shape. In winter 2011 ATLAS published 36 a table of their $95 \%$ CL upper limits on the cross section for dijet resonances with measured Gaussian widths of $3 \%, 5 \%, 7 \%, 10 \%$ and $15 \%$ of the peak mass. In summer $2011 \stackrel{38}{38}$ ATLAS 


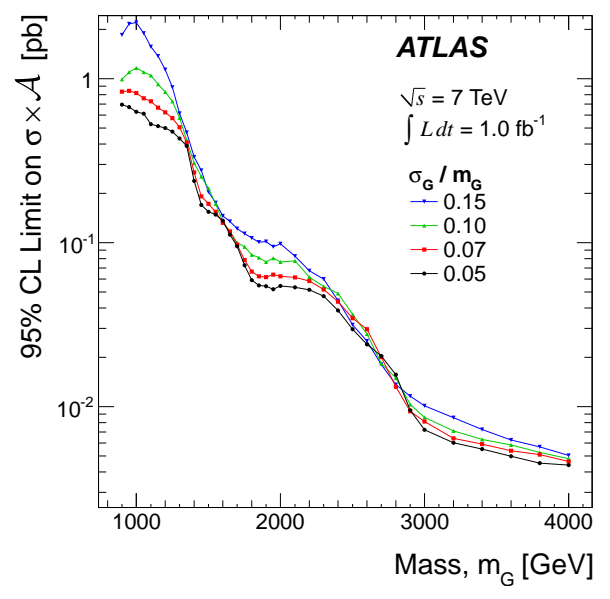

Fig. 21. Model independent limits from ATLAS in summer 2011. Observed upper limits at $95 \%$ CL for different Gaussian widths, from Ref. 38

updated all the limits, except they dropped the $3 \%$ Gaussian width, and published them in a table and also in Fig. 21. Notice that the resonances in Fig. 21 are not narrow, and the upper limits on the cross section increases as the Gaussian width of these resonance increases. In summer 2011 ATLAS published detailed instructions for how to obtain mass limits on new particles from these generic upper limits. In short, they recommended the production of a Monte Carlo sample for the calculation of the acceptance at parton level, and for the smearing of a dijet resonance signal with the ATLAS dijet resolution, which should then be truncated within $\pm 20 \%$ of the resonance peak before summing to find the total cross section. This is the first publication of generic limits on wide resonances since the 1993 publication from $\mathrm{CDF} 28$.

While this review is only covering searches in the dijet mass spectrum, we note that in 2011 ATLAS published the only search for dijet resonances at hadron colliders using the dijet angular distribution 36, and used it to set limits on excited quarks. The excited quark limits that we quote for ATLAS in table 4 and table 5 are from the search in the dijet mass spectrum only, not the search in the dijet angular distribution.

CMS published limits in $2010 \stackrel{35}{35}$ using $2.9 \mathrm{pb}^{-1}$ and in 201137 using $1 \mathrm{fb}^{-1}$. As for CDF, D0, and ATLAS, the handling of statistical uncertainties in the limits used a Bayesian procedure with uniform prior on the cross section. Systematic uncertainties considered were on the jet energy scale (10\% in 2010 and $2.2 \%$ in 2011), jet energy resolution (10\% producing negligile effect), luminosity (11\% in $2010,6 \%$ in 2011), and the background. The background parameterization used was Eq.49, the same as used by CDF in 2009 and ATLAS. The uncertainty on the background was determined in 2010 by varying the choice of the parameterization similar to CDF 

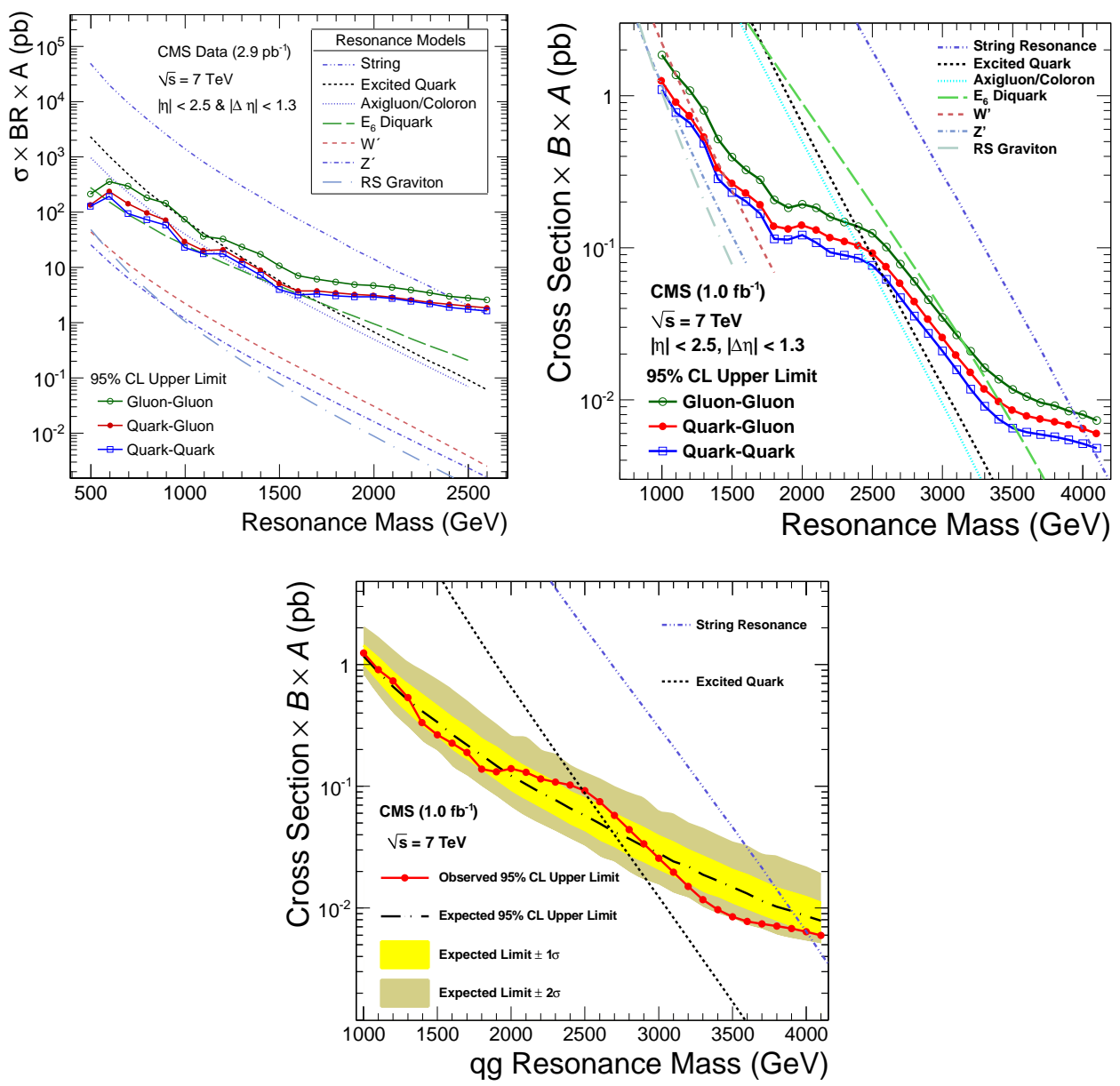

Fig. 22. Limits from CMS in 2010 and 2011. (top left) ) Observed upper limits at 95\% CL on dijet resonance cross section times branching ratio times acceptance using $2.9 \mathrm{nb}^{-1}$, shown separately for gluon-gluon, quark-gluon, and quark-quark resonances, and compared with predictions from various models. From Ref. 35. Copyright 2010 by the American Physical Society. (top right) Same, using $1.0 \mathrm{fb}^{-1}$ and (bottom) same for only quark-gluon resonances and including expected limits and the $1 \sigma$ and $2 \sigma$ variations in the expected limits, from Ref. 37. Copyright 2011, with permission from Elsevier.

in 1997, and in 2011 by varying the fit parameters within their statistical uncertainty, similar to ATLAS. The procedure for incorporating systematic uncertainties used by CMS in 2010 was the same as the method employed by CDF in 1997, and was described as "an approximate technique, which in our application is generally more conservative than a fully Bayesian treatment". In 2010 the systematic uncertainties increased the cross-section upper limits by $17 \%-49 \%$. In 2011 CMS used a fully Bayesian procedure with Gaussian priors for incorporating systematics. The 
updated procedure, along with the reduced systematics on the jet energy scale, had a much smaller effect on the observed cross-section upper limits than in 2010.

CMS used the shapes of narrow $q q, q g$ and $g g$ resonances to set generic upper limits at 95\% CL on the cross section times branching ratio into dijets times acceptance, and published the numbers in tables in both 2010 and 2011. In Fig. 22 the generic upper limits are compared to the dijet decays of string resonances, excited quarks, axigluons, colorons, $E_{6}$ diquarks, $W^{\prime}, Z^{\prime}$, and Randall-Sundrum gravitons. The model cross sections were LO calculations using CTEQ6L parton distributions, the same calculation as at CDF in 1997, and included the same "K-factors" for $W^{\prime}$ and $Z^{\prime}$ 54. The calculations for axigluons, $W^{\prime}, Z^{\prime}$, and $E_{6}$ diquarks employed the narrow width approximation discussed in section 2.2.11. The CMS axigluon calculation was very different from the calculation done by ATLAS, and the CMS $q^{*}$ calculation was slightly different than ATLAS, as discussed in Appendix A. The excluded regions for all models are shown in table 4 and the expected and observed limits are compared with ATLAS in table 5 . The differences for a data sample of 1 $\mathrm{fb}^{-1}$ are discussed in the next paragraph. In 2011, CMS also measured the $1 \sigma$ and $2 \sigma$ variations in the expected limit, and showed these for $q g$ resonances in Fig. 22 The largest upward fluctuation in the 2011 data was near $2.5 \mathrm{TeV}$ which caused the observed limit to be less than the expected limit for both excited quarks and axigluons. In 2011 CMS published the most stringent limits to date on string resonances, $E_{6}$ diquarks, and the dijet decays of $W^{\prime}$.

Table 5. Expected and observed mass limits at ATLAS and CMS from searches in the dijet mass spectrum.

\begin{tabular}{lllcccc}
\hline Expt. & Year & $\begin{array}{l}\int L d t \\
\left(p b^{-1}\right)\end{array}$ & $\begin{array}{c}\text { Axigluon } \\
\text { Expected } \\
(\mathrm{TeV})\end{array}$ & $\begin{array}{c}\text { Excited Quark } \\
(\mathrm{TeV})\end{array}$ & $\begin{array}{c}\text { Expected } \\
(\mathrm{TeV})\end{array}$ & $\begin{array}{c}\text { Observed } \\
(\mathrm{TeV})\end{array}$ \\
\hline ATLAS & 2010 & $3.2 \times 10^{-1}$ & - & - & 1.06 & 1.26 \\
CMS & 2010 & $2.9 \times 10^{0}$ & 1.32 & 1.58 & 1.23 & 1.17 \\
ATLAS & $2011 \mathrm{w}$ & $3.6 \times 10^{1}$ & 2.01 & 2.10 & 2.07 & 2.15 \\
CMS & 2011 & $1.0 \times 10^{3}$ & 2.66 & 2.47 & 2.68 & 2.49 \\
ATLAS & $2011 \mathrm{~s}$ & $1.0 \times 10^{3}$ & 3.07 & 3.32 & 2.81 & 2.99 \\
\hline
\end{tabular}

The CMS publication in 2011 37 and the ATLAS publication in summer 201136, both using an integrated luminosity of $1 \mathrm{fb}^{-1}$, reported different upper limits for the same model as shown in table 5 . The greatest difference lies in the observed limits for axigluons (CMS 2.47 TeV, ATLAS $3.32 \mathrm{TeV}$ ) but there is also a significant difference for excited quarks (CMS 2.49 TeV, ATLAS 2.99 TeV). As noted above, the observed limits by ATLAS were greater than their expected limits due to a downward fluctuation in the data, and the observed limits by CMS were less than the expected limits due to an upward fluctuation in the data, so these fluctuations clearly contributed to the difference in the observed limits between the two experiments. The fairest comparison should be in the expected limits. However, 
there is still a significant difference in the expected mass limits for axigluons (CMS $2.66 \mathrm{TeV}$, ATLAS 3.07 TeV) and a smaller but non-negligible difference in the expected mass limits for excited quarks (CMS $2.68 \mathrm{TeV}$, ATLAS $2.81 \mathrm{TeV}$ ). For both models, the vast majority of the difference in the mass limits appears to result in differences in the calculated cross section of the model, which we discuss in detail in Appendix A.

The most direct and natural comparison between the performance of ATLAS and CMS is in their expected limits on the cross section, as opposed to their expected mass limits on models. At a resonance mass of $3 \mathrm{TeV}$, the ATLAS expected upper limit on cross section time acceptance for excited quarks is $0.023 \mathrm{pb}$, while the CMS expected upper limit on cross section times acceptance for $q g$ resonances is 0.028 pb. For excited quark signals at high masses the CMS acceptance is $14 \%$ greater than the ATLAS acceptance. After correcting for acceptance the ATLAS expected limit on the cross section is about $10 \%$ more stringent than the CMS limit, which would give an expected mass limit about $1 \%$ more stringent. Compared this way the ATLAS and CMS performance is similar. This is expected given the comparable capabilities of the two experiments with the same integrated luminosity at the same center-of-mass energy.

\section{Summary}

Dijet resonance searches at hadron colliders have constrained a rich variety of models of new physics. Limits have been set on models motivated by grand unification, string theory, technicolor, compositeness, and ideas for new color interactions. Mass limits on the majority of models constrained are summarized in table 4. Limits on many colored resonances, like axigluons, colorons, and excited quarks, are now around $3 \mathrm{TeV}$ and limits as large as $4 \mathrm{TeV}$ have been set. In addition to model specific limits, the searches have provided model independent limits on the cross section for dijet resonances that can be used to constrain future models of new particle production.

The most important factors influencing the sensitivity of dijet resonance searches are the center-of-mass energy of the collider and the integrated luminosity of the dataset. The largest experimental uncertainty, the jet energy scale, now contributes little to upper limits on the cross section. Nevertheless, the experiments must remain vigilant that uncertainties in the jet energy scale do not manufacture a dijet resonance signal or hide one. Uncertainties in jet resolution at the experiments have usually had negligible effect. The searches are always dominated by statistical uncertainties, and the experiments have therefore for the most part done the most important thing, which is to rapidly search and publish when the energy or integrated luminosity have increased significantly. Keeping the search simple and model independent has made this easier.

In the course of time, the techniques and ideas employed by the experiments have varied significantly. However, an evolution can be observed towards standard 
practices, and it is worth noting the most important ones which may be useful for future searches as well.

The existence of a permanent record of fluctuations in the data for future reference, is helpful when experiments publish detailed comparisons of their dijet mass data to the background prediction. This was attempted by CDF and D0 with a ratio plot, but the plot of the bin-by-bin significance of the difference between data and background, introduced by the ATLAS collaboration and adopted by CMS, is ideal for this purpose. Estimations of the global significance of fluctuations introduced by ATLAS are instructive, and it would be even more helpful if they were accompanied by the undiluted estimates of the local significance of the fluctuation.

Both the ATLAS and CMS experiments have adopted a parametrization from CDF to model their QCD background. This is because a parameterization fit to the data generally gives a better model of the background than QCD calculations.

The experimental practice of reporting cross-section upper limits on dijet resonances, in addition to mass limits on specific models, has been important to allow continued use of the data to constrain models (see e.g. Ref 13). We have also shown that the cross section limits are important to understand and compare the results of the experiments,because differences in the calculation of the model cross sections often affect the mass limits significantly, as we saw in the comparison of the CDF and D0 limits with $100 \mathrm{pb}^{-1}$ and the ATLAS and CMS limits with $1 \mathrm{fb}^{-1}$. Clearly it would also be helpful if the experiments used a common method of calculating the model cross sections.

Finally, to understand the results of the experiments and to compare them, it has been important to have expected upper limits accompanied by their statistical variations, as well as observed upper limits. This practice was introduced by ATLAS and adopted by CMS, and is a visible benefit of the modern statistical techniques that are now commonly employed in the searches.

We look forward to future searches for dijet resonances at hadron colliders and their discovery of new physics beyond the standard model.

\section{Acknowledgments}

We gratefully acknowledge discussions with our colleagues who conducted dijet resonance searches at hadron colliders. In particular, to compare CMS and ATLAS, critical information was provided by Georgios Choudalakis and Alfonso Zerwekh from ATLAS, and Maxime Gouzevitch and Chiyoung Jeong from CMS. For sharing information and useful discussions over the years, we also thank Iain Bertram, John Paul Chou, Kenichi Hatakeyama, Emilio Meschi, Maurizio Pierini, Sertac Ozturk and Pierre Savard. For assistance with calculations and concepts of the models over the years we thank our colleagues in the theory community, including Uli Baur, Estia Eichten, Ken Lane, Can Kilic, Steve Mrenna, Tom Rizzo and Scott Thomas. This research was supported by Fermilab, operated by Fermi Research Alliance, LLC under Contract No. De-AC02-07CH11359 with the United States Department 
of Energy.

\section{Appendix A. Axigluon and Excited Quark Calculations from ATLAS and CMS}

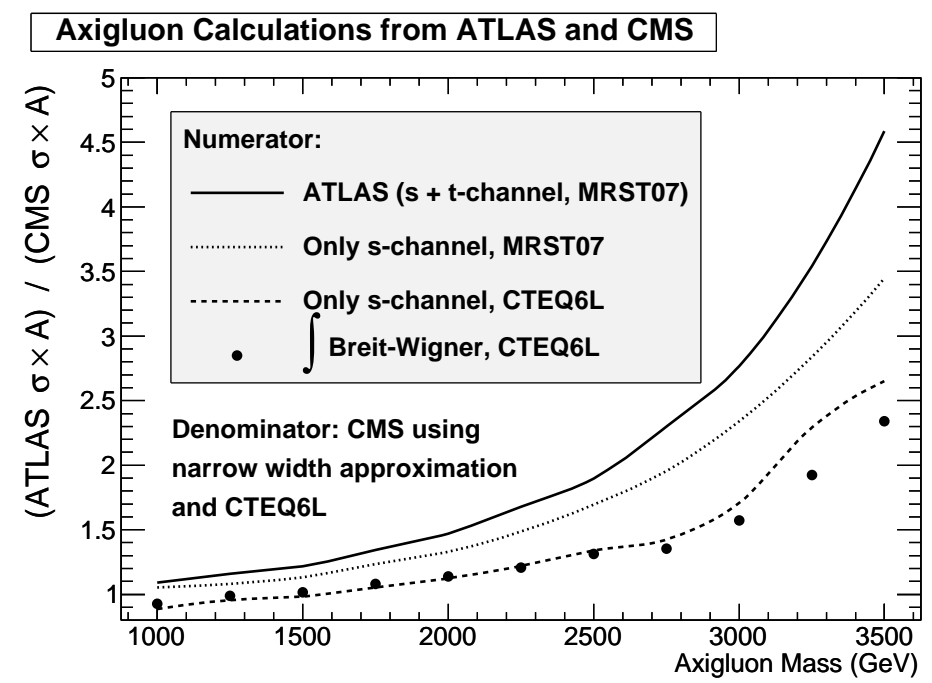

Fig. 23. Ratio of the ATLAS and CMS calculations of the axigluon cross section times branching ratio times acceptance.

Fig. 23 shows the ratio ATLAS / CMS for the calculated cross section times acceptance for axigluons decaying to dijets in each experiment. The solid curve in Fig. 23 shows the ratio of the cross section times acceptance curves published by the two experiments in Fig. 20 and Fig. 22 for $1 \mathrm{fb}^{-1}$. At an axigluon mass of $1 \mathrm{TeV}$ the calculations agree, and the ratio is the relative acceptance of the two experiments, but the ratio increases rapidly with mass. We will use a $3 \mathrm{TeV}$ axigluon resonance as an example, where the ratio of the cross section times acceptance published by ATLAS and CMS is a factor of 2.8. The Axigluon models employed by the two experiments are the same but there are differences in the way the two experiments perform the cross section calculations. The major sources of difference are

(i) Resonance tail. CMS uses the narrow width approximation which accounts for only the cross section at the resonant pole, while ATLAS uses CALCHEP and integrates the cross section over a dijet mass window within $\pm 30 \%$ of the resonance pole $(0.7 M<m<1.3 M)$ picking up a large tail at masses beneath the pole. The affect of ATLAS integration over the mass window is shown by both a CALCHEP calculation 61 (dashed curve in Fig. 23) and by our own calculation of the affect of integrating a Breit-Wigner on the CMS calculation 
(points in Fig.23). After taking out differences due to acceptance, this increases the ATLAS cross section by a factor of 1.92 for a $3 \mathrm{TeV}$ axigluon according to the CALCHEP calculation.

(ii) Parton distributions. CMS uses CTEQ6L and ATLAS uses MRST07. Comparing the dashed and dotted curves in Fig. 23 which come from a CALCHEP calculation 61 , we find this increases the ATLAS cross section by a factor of 1.37 for a $3 \mathrm{TeV}$ axigluon.

(iii) Processes included. CMS includes only the process where axigluons are produced resonantly in the s-channel, while ATLAS also includes processes where

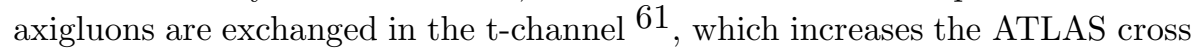
section by a factor of 1.18 for a $3 \mathrm{TeV}$ axigluon.

(iv) Acceptance. The acceptance of the two experiments for Axigluons is dominated by the $\left|\cos \theta^{*}\right|$ cut which is 0.57 for CMS and 0.54 for ATLAS. The combined affect of this cut and the ATLAS removal of a narrow region of the detector decreases the ATLAS acceptance by a factor of 0.89 .

The differences in the axigluon cross section calculations at ATLAS and CMS are the largest source of the difference in the expected limits and requires further discussion. The resonance tail at low mass is the largest single effect. How to handle its contribution to the new particle cross section, is a common problem faced by every resonance search in hadron colliders. It is caused by the decrease of the parton distributions of the proton with increasing fractional momentum $x \sim m / \sqrt{s}$, and is a particularly severe problem for $q \bar{q}$ resonances at $p p$ colliders. The narrow width approximation used by CMS, and discussed in section 2.2.11, is commonly employed by theorists to calculate the cross section for new particles. It replaces the true shape of the resonance with a delta function at the resonance pole, and therefore matches well the experimental approximation of both the ATLAS and CMS experiments when employing narrow resonance shapes to search for new physics and set limits on the cross section. However, the narrow width approximation clearly underestimates the true axigluon cross section, a significant fraction of which is off the pole at lower masses. The ATLAS choice to integrate over the actual line shape gives a total axigluon cross section that may be more realistic. However, this may not match as well the assumption of a narrow resonance shape peaked at the pole which was used to set cross section limits. The ATLAS choice to include t-channel processes in the total cross section again gives a result that may be more realistic than the CMS choice to only include s-channel processes. However, it is unclear whether the ATLAS and CMS methodology of fitting the data for the background shape and normalization effectively absorbs some of the cross section of these additional processes into the background, setting limits on only the bump like component of the resonances. The same can be said for the remainder of the axigluon tail at masses outside the search window which is ignored by both experiments. Further, both experiments ignore coherence between QCD and axigluons, which may significantly affect the tails of the distribution, and is likely an issue when the tail has a larger 
cross section than the peak. Finally we note that the different choice of PDFs by the two experiment is a source of variation in the mass limits that is easy to remove in the future.

A similar comparison between the model cross sections for excited quarks has been done at CMS and ATLAS. Here the calculated values of $(\sigma \times A)$ at ATLAS at a mass of $3 \mathrm{TeV}$ are only $16 \%$ greater than the reported values of $(\sigma \times B \times A)$ at CMS. There are three factors making up this difference:

(i) Parton distributions. CMS uses CTEQ6L and ATLAS uses MRST07, making the ATLAS cross section greater by about $10 \%$ at $\mathrm{M}=3 \mathrm{TeV}$.

(ii) Branching Fraction. CMS includes only $q^{*} \rightarrow q g$ decays in the dijet cross section, which make up $84 \%$ of the branching fraction. ATLAS also includes the $q W, q Z$ and $q \gamma$ decays. This choice increases the ATLAS cross section by a factor of roughly $1 / 0.84=20 \%$

(iii) Acceptance. The acceptance of the two experiments for excited quarks, which at resonance mass of $3 \mathrm{TeV}$ is 0.57 for CMS and 0.50 for ATLAS. The ATLAS acceptance is smaller by $12 \%$.

\section{References}

1. B. L. Combridge, J. Kripfganz and J. Ranft, Phys. Lett. B 70, 234, (1977).

2. R. K. Ellis, W. J. Stirling and B. R. Webber, Cambridge University Press, QCD and Collider Physics (1996).

3. U. Baur, I. Hinchliffe and D. Zeppenfeld, Int. J. Mod. Phys. A 2, 1285, (1987).

4. U. Baur, M. Spira and P. M. Zerwas, Phys. Rev. D 42, 815 (1990).

5. L. Randall and R. Sundrum, Phys. Rev. Lett. 83, 3370 (1999).

6. L. Randall and R. Sundrum, Phys. Rev. Lett. 83, 4690 (1999).

7. H. Davoudiasl, J. L. Hewett and T. G. Rizzo, Phys. Rev. Lett. 84, 2080 (2000).

8. J. Bijnens, P. Eerola, M. Maul, A. Mansson, T. Sjostrand, Phys. Lett. B 503,341 (2001).

9. P. H. Frampton and S. L. Glashow, Phys. Lett. B 190, 157 (1987).

10. R. S. Chivukula, A. G. Cohen and E. H. Simmons, Phys. Lett. B 380, 92, (1986).

11. E. H. Simmons, Phys. Rev. D 55, 1678 (1997).

12. J. Bagger, C. Schmidt and S. King, Phys. Rev. D 37, 1188 (1988).

13. T. Han, I. Lewis and Z. Liu, JHEP 12, 085 (2010).

14. E. Eichten, I. Hinchliffe, K. D. Lane and C. Quigg, Rev. Mod. Phys. 56, 579 (1984).

15. L. A. Anchordoqui et al., Phys. Rev. Lett. 101, 241803, (2008)

16. S. Cullen, M. Perelstein and M. E. Peskin, Phys. Rev. D 62, 055012, (2000).

17. P. Candelas, G. T. Horowitz, A. Strominger and E. Witten, Nucl. Phys. B 258, 46, (1985).

18. J. L. Hewett and T. G. Rizzo, Phys. Rept. 183, 193 (1989).

19. G. Katsilieris, O. Korakiantitis and S. Vlassopulos, Phys. Lett. B 288, 221, (1992).

20. V. D. Angelopoulos, John R. Ellis, H. Kowalski, D. V. Nanopoulos, N. D. Tracas, F. Zwirner, Nucl.Phys.B 292, 59, (1987).

21. B. Holdom, Phys. Rev. D 24, 1441 (1981); Phys. Lett.B 150, 301 (1985); T. Appelquist, D. Karabali and L. C. R. Wijewardhana, Phys. Rev. Lett. 57, 957 (1986); T. Appelquist and L. C. R. Wijewardhana, Phys. Rev. D 36, 568 (1987); K. Yamawaki, M. Bando and K. Matumoto, Phys. Rev. Lett. 56, 1335 (1986);T. Akiba and T. Yanagida, Phys. Lett. B 169, 432 (1986). 
22. K. Lane and M. Ramana, Phys. Rev. D 44, 2678 (1991).

23. K. Lane and S. Mrenna, Phys. Rev. D 67, 115011 (2003).

24. UA1 Collab. (G. Arnison et al.), Phys. Lett. B 172, 461 (1986).

25. UA1 Collab. (C. Albajar et al.), Phys. Lett. B 209, 127 (1988).

26. CDF Collab. (F. Abe et al.), Phys. Rev. D 41, 1722 (1990).

27. UA2 Collab. (J. Alitti et al.), Z. Phys. C 49,17 (1991).

28. CDF Collab. (F. Abe et al.), Phys. Rev. Lett. 71, 2542 (1993).

29. UA2 Collab. (J. Alitti et al.), Nucl. Phys. B400, 3 (1993).

30. CDF Collab. (F. Abe et al.), Phys. Rev. Lett. 74, 3538 (1995).

31. CDF Collab. (F. Abe et al.), Phys. Rev. D. 55, R5263 (1997).

32. D0 Collab. (V.M. Abazov et al.), Phys. Rev. D. 69, R111101 (2004).

33. CDF Collab. (T. Aaltonen et al.), Phys. Rev. D. 79, 112002 (2009).

34. ATLAS Collab. (G. Aad et al.), Phys. Rev. Lett. 105, 161801 (2010).

35. CMS Collab. (V. Khachatryan et al.), Phys. Rev. Lett. 105, 211801 (2010).

36. ATLAS Collab. (G. Aad et al.), New J. Phys. 13, 053044 (2011).

37. CMS Collab. (S. Chatrchyan et al.), Phys. Lett. B 704, 123 (2011).

38. ATLAS Collab. (G. Aad et al.), Submitted to Phys. Lett. B, arXiv:1108.6311.

39. F. Paige et al, arXiv:hep-ph/0312045

40. G. Corcella et al, JHEP 0101, 010 (2001) arXiv:hep-ph/0011363.

41. T. Sjostrand et al, Comput. Phys. Commun. 135, 238 (2001).

42. M. Gouzevitch from CMS Collaboration, private communication.

43. M. Cacciari, G.P. Salam, G. Soyez, JHEP 0804063 (2008); M. Cacciari and G.P. Salam, Phys. Lett. B 64157 (2006) hep-ph/0512210; M. Cacciari, G.P. Salam and G. Soyez, URL http://www.fastjet.fr

44. H. G. Gauch, Scientific Method in Practice, (Cambridge University Press, New York, 2003).

45. I. Bertram, arXiv:hep-ph/9811445v1.

46. CDF Collab. (F. Abe et al.),Phys. Rev. Lett.77, 438 (1996).

47. E. Gross and O. Vitells, Eur. Phys. J.C70, 525 (2010).

48. W. T. Giele, E.W.N. Glover, and D.A. Kosower, Phys. Rev. Lett. 73, 2019 (1994).

49. J. Pumplin et al., JHEP 07, 12 (2002).

50. T. Kluge, K. Rabbertz, and M. Wobisch, arXiv:hep-ph/0609285 2 .

51. D. Stump et al., JHEP 10, 46 (2003).

52. CDF Collab. (T. Aaltonen et al.) Phys. Rev. D79, 011101 (2009); G. Choudalakis, arXiv:physics.data-an/1101.0390.

53. CDF Collab. (F. Abe et al.),Phys. Rev. Lett.72, 3004 (1994).

54. K. Gumus et al., CMS sensitivity to dijet resonances, CMS Note 2006/070 (2006), URL http://cdsweb.cern.ch/record/962025

55. I. Bertram et al, "A Recipe for the Construction of Confidence Limits", FERMILABTM-2104 (2000).

56. L. Dermortier, in Proceedings of the Conference on Advanced Statistical Techniques in Particle Physics (Institute for Particle Physics Phenomenology, University of Durham, UK, 2002),p. 18.

57. ATLAS Collab., ATLAS Monte Carlo turnes for MC09, ATLAS Note ATL-PHYPUB-2010-002.

58. A. Sherstnev and R. S. Thorne, Eur. Phys. J. C. 55, 553 (2008).

59. A. Pukhov, arXiv:hep-ph/0412191.

60. J. Alwall et al., arXiv:hep-ph/1106.0522.

61. A. Zerwekh from ATLAS Collaboration, private communication. 